\title{
An experimental investigation into spatiotemporal intermittencies in turbulent channel flow close to transition
}

\author{
R. D. Whalley ${ }^{1}$ (1) D. J. C. Dennis ${ }^{2} \cdot$ M. D. Graham ${ }^{3} \cdot$ R. J. Poole ${ }^{2}$
}

Received: 16 October 2018 / Revised: 12 March 2019 / Accepted: 29 April 2019 / Published online: 27 May 2019

(C) The Author(s) 2019

\begin{abstract}
Experiments are presented to characterize low- and high-drag turbulence events in channel flows close to transition, which last for a certain duration, at friction Reynolds numbers ranging from $R e_{\tau}=70-100$. The spatiotemporal intermittencies are identified by applying conditional sampling techniques to simultaneously acquired wall shear stress and velocity data using either single-point laser Doppler velocimetry (LDV) or stereoscopic particle image velocimetry (SPIV). It is shown that ensemble-averaged streamwise velocity during intervals of low drag fall close to a recently discovered exact coherent state (ECS), in agreement with recent direct numerical simulation (DNS) results. The low-drag intervals are characterized by a low-stress streak which is flanked on either side by a streamwise vortex, forming a counter-rotating vortex pair. Ensemble-averaged streamwise velocity during intervals of high drag fall further below the Prandtl-von Kármán log-law with increasing Reynolds number. Generally, higher levels of turbulence intensity are observed during intervals of low drag when compared to similar intervals of high drag.
\end{abstract}

\section{Graphical abstract}

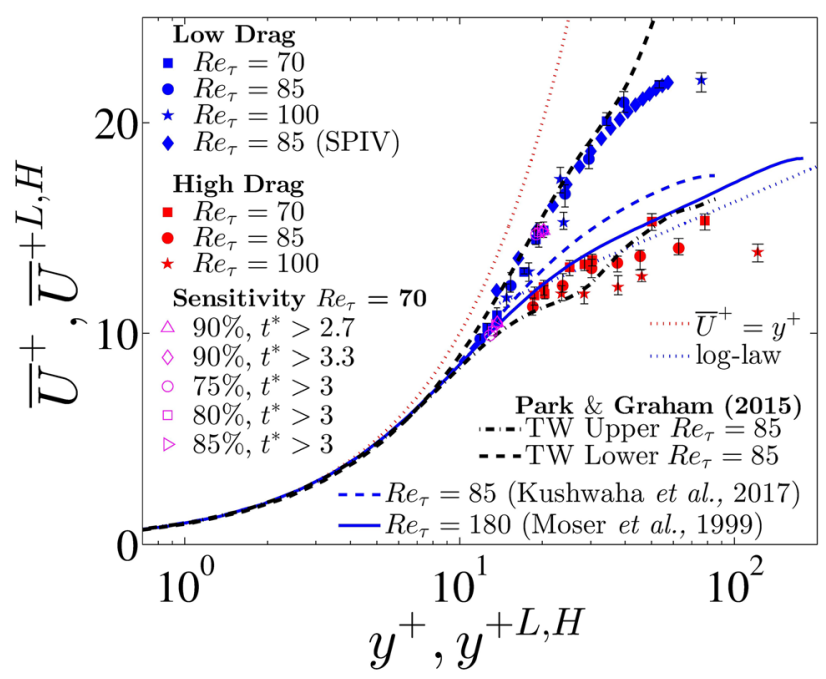

R. D. Whalley

Richard.Whalley@newcastle.ac.uk

1 School of Engineering, Newcastle University, Newcastle upon Tyne NE1 7RU, UK

2 School of Engineering, University of Liverpool, Liverpool L69 3GH, UK

3 Department of Chemical and Biological Engineering, University of Wisconsin-Madison, Madison, WI 53706, USA

\section{Introduction}

Wall-turbulent flows can be visualized as a complex entanglement of quasi-streamwise vortices whose legs extend down towards the wall and induce the classical meandering near-wall streaky flow structure (Kline et al. 1967; Head and Bandyopadhyay 1981; Robinson 1991; Hutchins et al. 2005; 
Adrian 2007). Many proposed approaches for skin-friction reduction often focus on manipulation of the flow in the near-wall region through topography, suction and blowing or imposition of wall motions (Choi 1989; Karniadakis and Choi 2003; Hof et al. 2010; Choi et al. 2011; Garcia-Mayoral and Jimenez 2011; Quadrio 2011; Whalley and Choi 2014). But perhaps the most successful turbulence control technique, and one which can only be used in liquid flows, is the dissolution of rheology-modifying additives such as longchain polymers or worm-like surfactants to the working fluid (Virk 1975; Graham 2014; Owolabi et al. 2017).

Additives can significantly reduce the energy consumption in turbulent flow processes at least up to a certain level, even at high Reynolds numbers, giving rise to a phenomenon known as maximum-drag-reduction (MDR) (Virk 1975). Within the last 10 years, evidence from DNS studies has emerged that suggests that drag reduction in polymer solutions is connected to weakly turbulent flow structures found in Newtonian fluid flow that are unmasked as polymer viscoelasticity suppresses the dominant turbulent motions (Xi and Graham 2010a, b, 2012a, b; Wang et al. 2017). These computational studies, performed at low Reynolds number close to transition to turbulence, revealed intervals where the flow becomes relatively quiescent containing little drag, as had, in fact, been observed long ago (Jimenez and Moin 1991). These low-drag events were called intervals of "hibernating" turbulence and displayed a streamwise velocity profile similar to that found in polymer solutions at a substantial level of drag reduction.

Experimental evidence supporting the existence of the hibernating turbulence phenomena has recently been obtained. Whalley et al. (2017) showed that the hibernating turbulence flow structure in Newtonian fluid flow resembled a recently discovered traveling-wave solution (Park and Graham 2015), presenting a possible connection between the turbulent flows of polymeric solutions, transition to turbulence and nontrivial invariant solutions to the Navier-Stokes equations. Within the last 15 years, there has been a significant improvement in the physical understanding of transition to turbulence in wall-bounded turbulent flows (Hof et al. 2004; Kerswell 2005; Eckhardt et al. 2007; Willis et al. 2008; Kawahara et al. 2012), gained in part by building on the mathematical framework of dynamical systems theory. Of particular importance is the discovery of three-dimensional nonlinear traveling waves (TWs) to the Navier-Stokes equations (Waleffe 2003; Hof et al. 2004; Wedin and Kerswell 2004; Eckhardt et al. 2008; Gibson et al. 2008). These have allowed a priori investigation of the self-sustaining near-wall coherent structures observed in wall-turbulent flows. These solutions, or ECSs (Waleffe 1998), appear as a low-speed near-wall streak straddled by a counter-rotating vortex pair. How frequently Newtonian fluid flow enters into a state of low drag beyond transitional Reynolds numbers remains an open question; however, high-Reynolds-number turbulent boundary layer flow investigations have simply shown an upshift of the Prandtl-von Kármán log-law during intervals of low drag, rather than a change in slope (Hutchins et al. 2011).

In the present paper, the conditional sampling techniques used by Whalley et al. (2017) are extended and applied on simultaneously acquired wall shear stress and velocity data to further elucidate on the conditionally sampled instantaneous, and conditionally averaged turbulent trajectories of wall shear stresses and streamwise velocities during intervals of low drag in channel flows close to transition to turbulence. In addition, similar conditional sampling techniques are applied to the same simultaneously acquired wall shear stress and velocity data to explore the counterpart of hibernating turbulence, namely intervals of high drag.

\section{Experimental rig and instrumentation}

A schematic representation of the experimental facility used throughout this investigation is shown in Fig. 1. The flow loop incorporated a rectangular duct which had a width $(w)$
Fig. 1 Schematic representation of the channel flow facility. Flow is clockwise

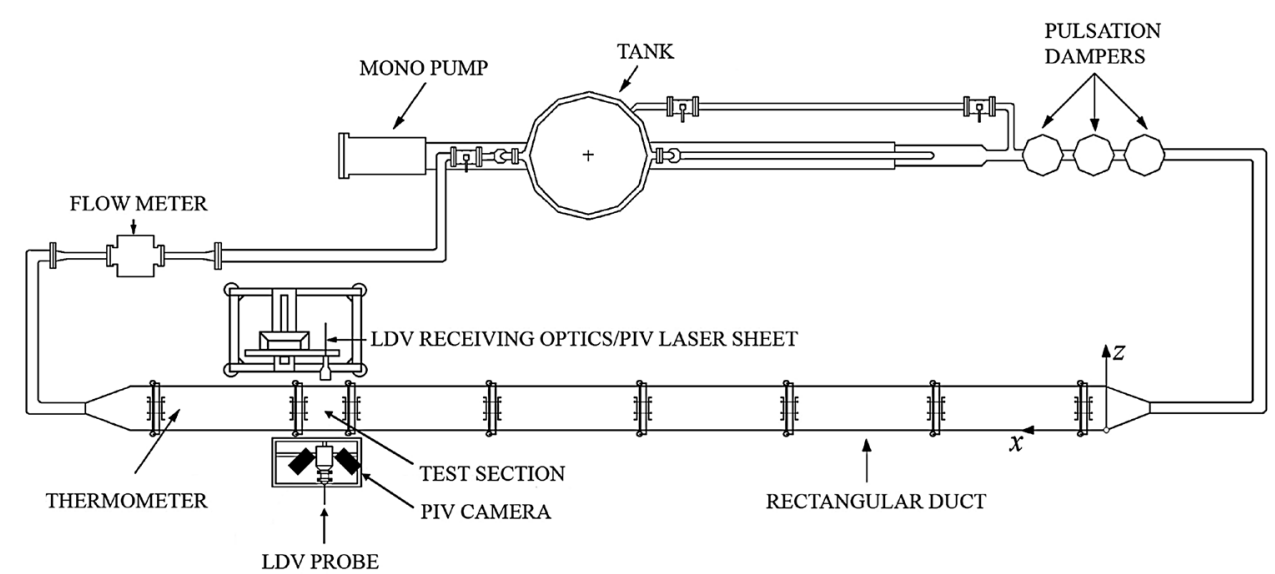


of $298 \mathrm{~mm}$, a half-height $(h)$ of $12.5 \mathrm{~mm}$, giving an aspect ratio $(w / h)$ of 23.84 , and was $7.45 \mathrm{~m}$ long. The rectangular duct consisted of six stainless steel modules and a test section. The stainless steel modules were $1.2 \mathrm{~m}$ long, machined and ground to size, and assembled with O-rings, screws and a bonding agent. Five of the stainless steel modules were upstream of the test section, which was $0.25 \mathrm{~m}$ long and had sidewalls fabricated with borosilicate glass to permit velocity measurements with LDV and SPIV. The Newtonian fluid used throughout this study was a nominal $60 / 40$ per cent concentration by weight of glycerine/water. The density was calculated by weighing a known volume of the solution and the kinematic viscosity was measured by a Cannon-Fenske viscometer. The fluid was circulated around the flow loop by a Mono type E101 progressive cavity pump via a stainless steel header tank. The mass flow rate was measured by an Endress and Hauser Promag P electromagnetic flowmeter and the ambient fluid temperature was monitored by a platinum resistance thermometer (PRT). The PRT was mounted in the final module of the rectangular duct and was powered by an Agilent 34970A switch unit, which provided temperature readings with a resolution of $0.01^{\circ} \mathrm{C}$ to an accuracy of $\pm 0.09^{\circ} \mathrm{C}$. Three pulsation dampers were located immediately downstream of the pump outlet to diminish any disturbances prior to entry into the rectangular duct. Fluid entered and left the rectangular duct through transition sections, which varied in cross section from circular to rectangular and from rectangular to circular, at the inlet and outlet, respectively. The flow was allowed to develop naturally, without the use of a turbulent trip, into a fully developed turbulent regime over the entire length of the duct, a distance of 596 channel half-heights.

Throughout the paper, $x, y$ and $z$ denote the streamwise, wall-normal and spanwise directions, and the symbols $U, V$ and $W$ indicate the instantaneous streamwise, wall-normal and spanwise velocities, respectively. The superscripts $L$ and $H$ indicate conditional sampling during low- and highdrag intervals, respectively, and an overbar indicates a timeaveraged or an ensemble-averaged quantity (e.g. $\bar{U}, \bar{U}^{L}$ ). Fluctuating velocities are represented by lowercase symbols (e.g. $u$ ) and primed symbols (e.g. $u^{\prime}$ ) denote r.m.s values of these fluctuations.

To detect low- and high-drag intervals, a Dantec 55R46 flush-mounted miniaturized hot-film probe was calibrated to measure the instantaneous wall shear stress of the turbulent channel flow. The probe was powered by a StreamLine Pro velocimetry system and balanced via a $91 \mathrm{C} 10$ bridge module. The probe was operated in constant temperature mode with an overheat ratio of 1.08 . A gain of 16 was applied to the voltage signal, which was low-pass filtered at $1 \mathrm{kHz}$ prior to sampling. Once optimized by a square wave test, the probe had a typical frequency response of $\approx 10$ $\mathrm{kHz}$. The probe had a sensing element which was $0.2 \mathrm{~mm}$ long $\left(x^{+}=x \overline{u_{\tau}} / \nu=1.38\right)$ and $0.75 \mathrm{~mm}$ wide $\left(z^{+}=5.19\right)$ and was flush mounted on the lower wall of the channel at $x / h=488, z / w=0.33$ during the LDV measurements and at $x / h=415, z / w=0.5$ during the SPIV measurements. Here, $u_{\tau}=\left(\tau_{w} / \rho\right)^{1 / 2}$ is the friction velocity at $R e_{\tau}=\overline{u_{\tau}} h / v=85$, $\tau_{w}$ is the wall shear stress, $\rho$ is the fluid density and $v$ is the kinematic viscosity, $x / h=0$ is the location of the duct inlet and $z / w=0$ is the location of a side wall: see Fig. 1 for details of the co-ordinate system, with $y=0$ being the location of the lower wall.

The hot-film probe was calibrated in situ in the channel flow facility by a Druck LPX-9381 low-differential pressure transducer. The pressure transducer estimated the streamwise pressure gradient, from which the time-averaged wall shear stress could be determined, by measuring the difference in pressure across pressure taps installed on the lower wall of the stainless steel modules of the rectangular duct, over a distance of $2.05 \mathrm{~m}(x / h=164)$. The pressure transducer had a working range of $50 \mathrm{mbar}$, was accurate to \pm 0.05 mbar and was periodically calibrated against an MKS Baratron differential pressure transducer (1000 torr fsd). During the course of an experiment, the ambient fluid temperature would rise by typically $1^{\circ} \mathrm{C}$ due to the viscous heating of the pump. Since hot-film velocimetry is sensitive to temperature drift, the hot-film probe was calibrated before and after each experiment. The temperature during each experiment was carefully monitored by the PRT so that the voltage output from the hot-film probe could be linearly interpolated between the two calibration curves accordingly. Examples of the calibration curves, before and after an experiment at $\operatorname{Re}_{\tau}=70$, are shown in Fig. 2 and are fit with a third

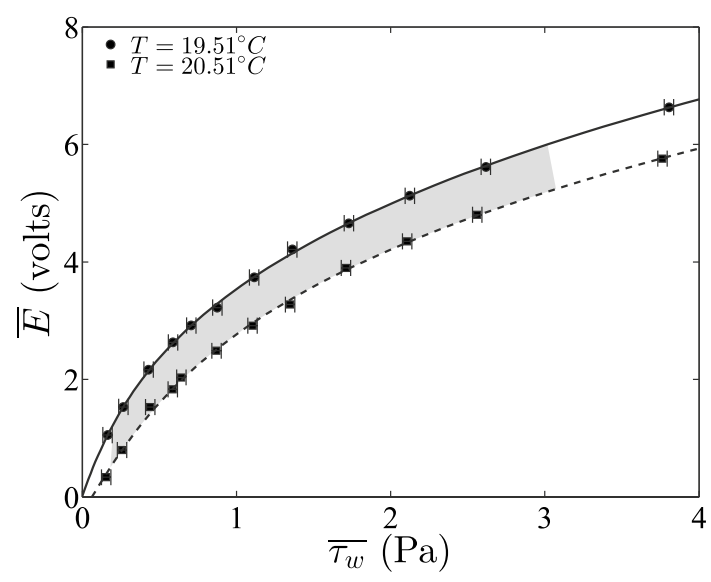

Fig. 2 Example of hot-film probe calibration curves before and after an experiment at $R e_{\tau}=70$. Both calibration curves are fit with a third-order polynomial. The ambient fluid temperature before and after the experiment was $T=19.51^{\circ} \mathrm{C}$ (filled black circle, solid black line) and $T=20.51^{\circ} \mathrm{C}$ (filled black square, dashed black line), respectively. The error bars show the uncertainty of the pressure transducer. The grey region indicates the range of instantaneous wall shear stress measured by the hot-film probe during the experiment 
order polynomial. At each point of the calibration curve, 90,000 samples from the hot-film probe and pressure transducer were acquired for each time average. The error bars indicate the uncertainty in the pressure measurements. The grey region indicates the range of instantaneous wall shear stress measured by the hot-film probe during the experiment.

The changes in turbulence structure during the low- and high-drag intervals were quantified by measuring the streamwise $(U)$ velocity component of the turbulent channel flow with LDV. The Dantec FiberFlow LDV system consisted of a $300 \mathrm{~mW}$ continuous wave laser, a $60 X 40$ laser light transmitter, a $60 \times 10$ probe, a 55X12 beam expander, a 55X35 photomultiplier tube and a 16-bit F50 Burst Spectrum Analyser (BSA). The LDV system was operated in burst mode in conjunction with forward scatter optics. The specially designed optical head provided a focal length of $160 \mathrm{~mm}$ and focused the laser beams to create a measurement volume with diameter of $0.025 \mathrm{~mm}\left(x^{+}=0.17\right)$ and length of 0.1 $\mathrm{mm}\left(z^{+}=0.69\right)$ in the working fluid. A 4-channel, 12-bit, sample-and-hold analogue-to-digital converter (ADC), built into the F50 BSA, was used to record the voltage signals outputted from the hot-film probe, pressure transducer and flowmeter. The ADC was controlled by Dantec BSA Flow software and sampled the devices when a Doppler burst was detected. This setup ensured that the streamwise velocity and wall shear stress were sampled simultaneously and enabled the wall shear stress to be additionally determined from the velocity gradient at the wall. Hence, the mean wall shear stress used in the determination of the mean friction velocity for the usual time-averaged data was taken to be the mean of the value determined from pressure drop measurements and that determined from the near-wall velocity gradient.

Throughout this experimental investigation, the streamwise velocity was measured $12.5 \mathrm{~mm}(h)$ downstream of the flush-mounted hot-film probe $(z / w=0.33)$ at various distances normal to the wall with LDV. Typically during an experiment, 30,000 velocity realizations were acquired at each wall-normal location within the viscous sublayer $\left(y^{+}<5\right)$, which was $600 \mu \mathrm{m}$ to $900 \mu \mathrm{m}$ thick over $R e_{\tau}=70-100$. Outside of the viscous sublayer, 50,000 velocity realizations were acquired. Within the viscous sublayer region, streamwise velocity data were acquired from a nominal wall-normal distance of $y=300 \mu \mathrm{m}$ to $y=600 \mu \mathrm{m}$ in $100 \mu \mathrm{m}$ increments followed by further measurements at $y=800 \mu \mathrm{m}$ and $y=1000 \mu \mathrm{m}$. This provided four or five data points within the viscous sublayer depending on the Reynolds number. A least-squares linear fit to the timeaveraged streamwise velocity data within the viscous sublayer provided an estimate of the time-averaged wall shear stress and the true location of the wall (Hutchins and Choi 2002). Here, it should be emphasized that the time-averaged wall shear stress measured by the hot-film probe was always within 5 per cent of the value obtained by linear fitting the
LDV data within the viscous sublayer. Since the LDV system acquired data randomly, at uneven sampling times, the transit-time weighting technique was used to compute all time-averaged quantities (Tropea 1995). During each experiment, either one or two wall-normal locations were sampled for extremely long durations. For example, at $R e_{\tau}=70$ and $R e_{\tau}=85$, the flow was sampled for a duration of $3 \mathrm{~h}$, capturing circa 2,000,000 velocity realizations at each wall-normal location $\left(R e_{\tau}=70: y^{+}=15,16,20,23,25,41,63 ; R e_{\tau}=85\right.$ : $\left.y^{+}=15,19,25,30,37,51\right)$, where the data were subsequently conditionally sampled and then ensemble averaged. As the Reynolds number increased, the number of low- and high-drag events detected each hour reduced. Therefore, at $R e_{\tau}=100$, the sampling time was increased to $6 \mathrm{~h}$, capturing circa 4,500,000 velocity realizations at each wall-normal location $\left(y^{+}=19,23,30,38,101\right)$. Each low- and high-drag event lasted on the order of $1 \mathrm{~s}$ and was typically resolved by a few hundred velocity/wall shear stress data points.

In a separate set of experiments, the streamwise, wallnormal and spanwise velocity components were measured simultaneously in the $y-z$ plane of the turbulent channel flow with an SPIV system from TSI, which provided global velocity measurements to an accuracy of 3-5\% (Westerweel 1997). The SPIV system consisted of two Photron FastCamSA3 cameras and a New Wave Research Pegasus PIV laser. The SPIV measurements were taken at $x / h=415$, directly above the location of the flush-mounted wall shear stress probe. The instantaneous wall shear stress signal was time stamped alongside data collected by the SPIV system using a National Instruments Data Acquisition module. The instantaneous wall shear stress signal was processed "on the fly", and once a low-drag event was detected, the SPIV data were downloaded for post-processing. Silver-coated glass hollow spheres with a nominal diameter of $10 \mu \mathrm{m}$ were used to seed the flow. The SPIV data were obtained at a friction Reynolds number of $R e_{\tau}=85$ with a field of view of $25 \times 37.5 \mathrm{~mm}^{2}$ $(y / h=2, z / h=3)$. Image pairs were acquired at a frequency of $62.5 \mathrm{~Hz}\left(t^{*}=t \overline{u_{\tau}} / h=0.04\right)$ with the time delay between image pairs being $750 \mu \mathrm{s}\left(t^{*}=0.0018\right)$. Data processing was performed using a recursive cross-correlation algorithm to generate velocity vectors over a $32 \times 32$ pixel $^{2}$ interrogation area with $50 \%$ overlap, providing a spatial resolution of $0.5 \times 0.7 \mathrm{~mm}^{2}\left(y^{+}=3.46, z^{+}=4.84\right)$.

Kline and McClintock (1953) showed that if a measured quantity, $\phi$, is a function of independent variables, $X_{i}$, of the form

$\phi=X_{1}^{a} X_{2}^{b} X_{3}^{c} \ldots X_{M}^{m}$,

then the uncertainty $\epsilon \phi$ can be expressed as

$$
\frac{\epsilon \phi}{\phi}= \pm\left\{\left(a \frac{\epsilon X_{1}}{X_{1}}\right)^{2}+\left(b \frac{\epsilon X_{2}}{X_{2}}\right)^{2}+\cdots+\left(m \frac{\epsilon X_{M}}{X_{M}}\right)^{2}\right\}^{\frac{1}{2}}
$$


Given that the Druck LPX-9381 pressure transducer had a quoted accuracy of $\pm 0.05 \mathrm{mbar}$, and the precision machined channel flow facility ensured that the relative uncertainties in the channel half-height and the streamwise distance between pressure tappings were negligibly small, $\sim 0.15 \%$, the uncertainty in the wall shear stress made by pressure-drop measurements, was $\epsilon \tau_{w}= \pm 0.03 \mathrm{~Pa}$. Hence, the relative uncertainty in the mean wall shear stress was $\epsilon \tau_{w} / \overline{\tau_{w}}= \pm 3.5 \%, \pm 3 \%$ and $\pm 2 \%$ for $R e_{\tau}=70,85$ and 100 , respectively. Weighing a known volume of the glycerine/ water solution provided the density of the working fluid to within $\pm 1 \%$. Therefore, the relative uncertainties in the mean friction velocity were $\epsilon u_{\tau} / \overline{u_{\tau}}=0.5 \epsilon \tau_{w} / \overline{\tau_{w}}= \pm 1.75 \%$, $\pm 1.5 \%$ and $\pm 1 \%$ for $R e_{\tau}=70,85$ and 100 , respectively. The Cannon-Fenske viscometer slowly released a sample of the glycerine/water solution over a set vertical distance within a precision glass tube over typically $400 \mathrm{~s}$. Six samples were timed per experimental run, providing an estimate of the relative uncertainty in the kinematic viscosity measurements of $\epsilon v / v \approx 0.5 \%$. The LDV probe was traversed in the wall-normal direction to an accuracy of $\pm 3 \mu \mathrm{m}$. Close to the wall, for example at $y=300 \mu \mathrm{m}$ where the flow was first sampled, the relative uncertainty of $\epsilon y^{+} / y^{+}= \pm 2.1 \%, \pm 1.9 \%$ and $\pm 1.5 \%$ for $R e_{\tau}=70,85$ and 100, respectively. Beyond the viscous sublayer $\left(y^{+}>5\right)$, where the relative uncertainty in wall-normal positioning became increasingly small, $\epsilon y^{+} / y^{+} \approx \epsilon u_{\tau} / \overline{u_{\tau}}$. Similarly, $\epsilon t^{*} / t^{*} \approx \epsilon u_{\tau} / \overline{u_{\tau}}(\sim 1-2 \%)$, and $\epsilon t^{+} / t^{+} \approx 2 \epsilon u_{\tau} / \overline{u_{\tau}}(\sim 2-4 \%)$. Calibration experiments suggest that the LDV system had an accuracy of $1-2 \%$ in the mean velocity, providing relative uncertainties in the mean streamwise velocity of $\epsilon U^{+} / \bar{U}^{+} \approx 2-4 \%$. Combining the experimental uncertainties for the streamwise velocity and wall-normal positioning provides an estimate for the relative uncertainty in the mean wall shear stress determined by the near-wall velocity gradient technique of $\epsilon \tau_{w} / \overline{\tau_{w}} \approx 2-4 \%$, agreeing well with the uncertainty analysis of Hutchins and Choi (2002).

\section{Validation profiles}

Validation profiles of the channel flow facility were acquired prior to studying the spatiotemporal turbulence phenomena. Shown in Fig. 3 is friction factor $(f)$ versus Reynolds number $(R e)$ for laminar, transitional and turbulent channel flow. Here, $R e=2 h \overline{U_{b}} / \nu$, where $U_{b}$ is the bulk velocity through the channel determined from the mass flow meter. The data collapses to the laminar curve $f=11.7 / R e$ and to the empirical curve for smooth turbulent channel flow, $f=0.073 R e^{-0.25}$ (Dean 1978), with error bars showing the uncertainty in the pressure-drop measurements. Of particular interest is the region of transition between the laminar and turbulent flow regimes. In subsequent sections, results of

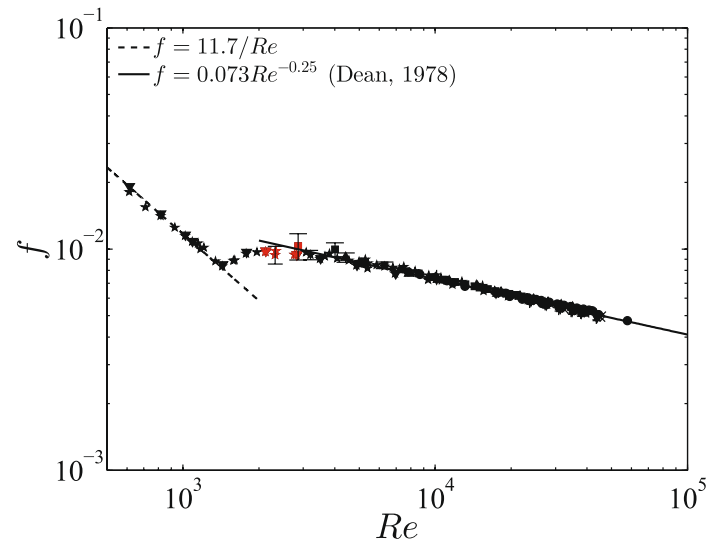

Fig. 3 Friction factor versus Reynolds number for laminar, transitional and turbulent channel flow. The plot comprises 15 data sets, acquired over 15 separate experiments, with the friction factor evaluated from mean pressure-drop measurements. The black dashed line is $f=11.7 / R e$ and the black solid line is $f=0.073 R^{-0.25}$ (Dean 1978). The red symbols highlight $R e=2000-3000$ $\left(R e_{\tau}=70-100\right)$, which is the Reynolds number range where the low- and high-drag events are detected in this investigation

conditionally sampled data will be presented at $R e_{\tau}=70,85$ and 100, which correspond to $R e=2000,2400$ and 3000, respectively. In this facility, sustained transition to turbulence is found to occur beyond $R e_{\tau}=46$ or $R e=1470$ (Alavyoon et al. 1986).

Figure 4a shows the time-averaged streamwise velocity $\bar{U}^{+}\left(=\bar{U} / \overline{u_{\tau}}\right)$ over the $R e_{\tau}$ range of interest. Data are acquired with LDV and SPIV and show excellent agreement with existing, very limited, low-Reynolds number experimental data, and with DNS data. As expected, the time-averaged streamwise velocity converges on the Prandtl-von Kármán log-law as the $R e_{\tau}$ increases (Wei and Willmarth 1989). Figure 4b shows turbulence intensity profiles for the three velocity components. The streamwise intensity captured with LDV (open symbols) shows excellent agreement when compared with existing lowReynolds number data. The SPIV data (closed symbols) have tended to slightly under-resolve the turbulent intensities in all three components. Given that the main component of velocity was through the laser light sheet, which intrinsically limits pixel displacement, and the entire channel height was visualized, the slight underestimation in intensities is not entirely unexpected (Christensen 2004; Hutchins et al. 2005). Overall, the SPIV data are in good agreement with existing DNS data. At $R e_{\tau}=85$, SPIV has slightly underestimated $u^{\prime+} \sim 8 \%$ and $w^{\prime+} \sim 5 \%$ for $y^{+}<40$, and $v^{\prime+} \sim 15 \%$ below the DNS data over the full channel half-height. At $R e_{\tau}=180, u^{\prime+} \sim 12 \%$ below the DNS data at $y^{+} \approx 30$, and converges on the DNS data as the channel centre line is approached. The wall-normal intensity, $v^{+} \sim 4 \%$ below the DNS data for $y^{+}<130$, and 

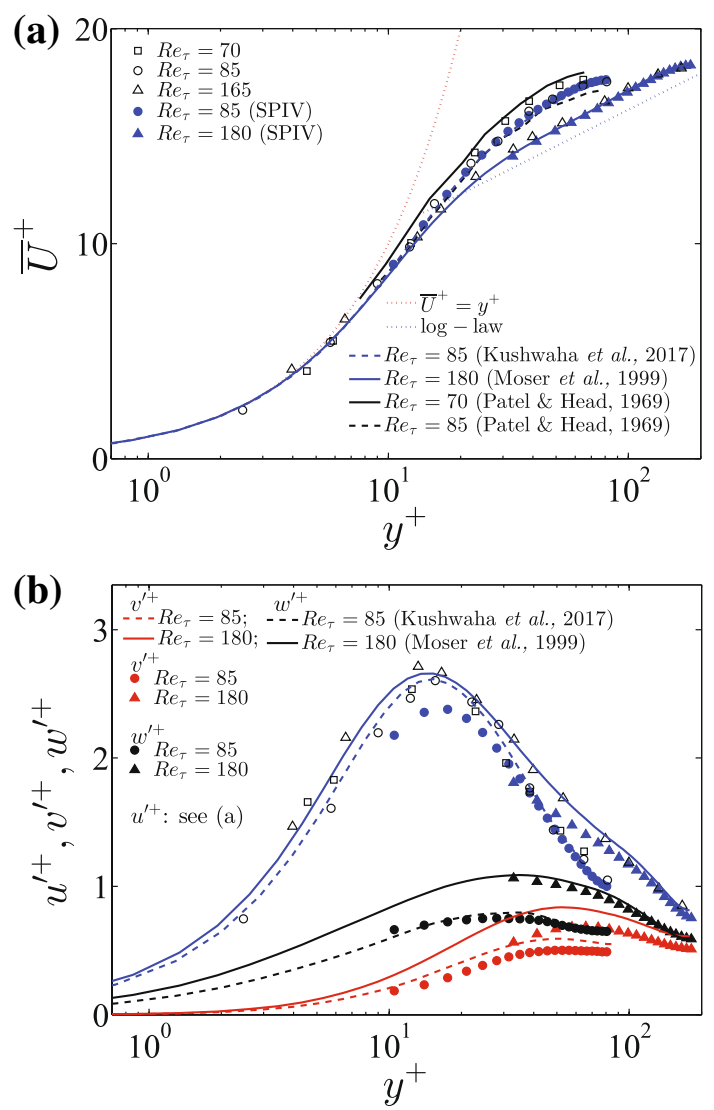

Fig. 4 a Time-averaged streamwise velocity scaled with time-averaged wall shear stress acquired with LDV at $R e_{\tau}=70$ (open square), $R e_{\tau}=85$ (open circle), $R e_{\tau}=165$ (open triangle) and with stereoscopic PIV at $R e_{\tau}=85$ (filled blue circle), $R e_{\tau}=180$ (filled blue triangle). The present experimental data are compared to DNS data at $R e_{\tau}=85$ [blue dashed line, Kushwaha et al. (2017)] and $R e_{\tau}=180$ [blue solid line, Moser et al. (1999)], and experimental data from Patel and Head (1969) at $R e_{\tau}=70$ (black solid line) and $R e_{\tau}=85$ (black dashed line). The red dotted line is the viscous sublayer profile: $\bar{U}^{+}=y^{+}$, blue dotted line is the Prandtl-von Kármán log-law: $\bar{U}^{+}=2.44 \ln y^{+}+5$. b Time-averaged turbulence intensities scaled with time-averaged wall shear stress. Streamwise turbulence intensity has the same symbols as in a. Wall-normal turbulence intensity ( $R e_{\tau}=85$ : filled red circle, $R e_{\tau}=180$ : filled red triangle) and spanwise turbulence intensity ( $R e_{\tau}=85$ : filled black circle, $R e_{\tau}=180$ : filled black triangle) are acquired with stereoscopic PIV and are compared to DNS data at $R e_{\tau}=85$ [red dashed line: $v^{\prime+}$; black dashed line: $w^{\prime+}$, Kushwaha et al. (2017)] and $\operatorname{Re}_{\tau}=180$ [red solid line: $v^{\prime+}$; black solid line: $w^{\prime+}$, Moser et al. (1999)]

$w^{\prime+}$ underestimated the DNS by $\sim 15 \%$ over the entire channel half-height.

Alfredsson et al. (1988) showed that the thermal conductivity of a fluid can effect a hot-film probe's ability to measure fluctuating wall shear stresses. Hot-film measurements in low thermal conductivity $(\lambda)$ fluids, such as air $(\lambda=0.024 \mathrm{~W} / \mathrm{mK})$ or oil $(\lambda=0.18 \mathrm{~W} / \mathrm{mK})$, underestimated the wall shear stress fluctuations by up to a factor of 4 ; however, measurements in water $(\lambda=0.60 \mathrm{~W} / \mathrm{mK})$ remained unaffected. The damping

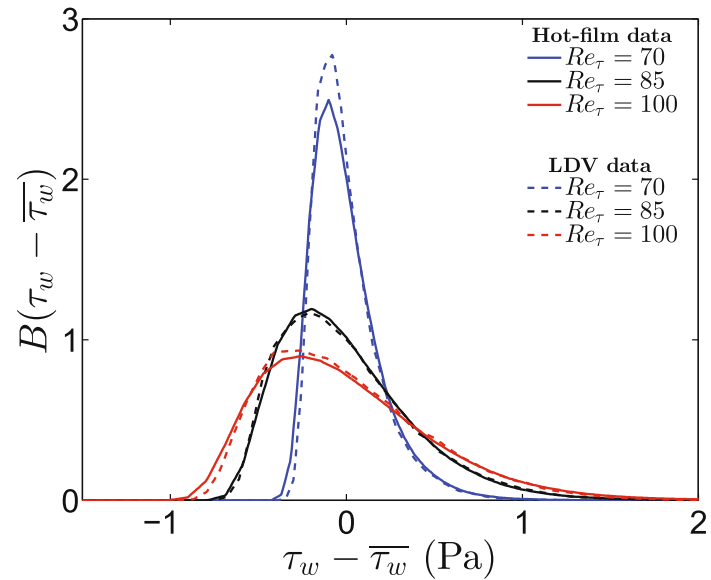

Fig. 5 PDFs of fluctuating wall shear stress acquired with LDV (dashed lines) and a hot-film probe (solid lines) at $R e_{\tau}=70$ (blue lines), $R e_{\tau}=85$ (black lines) and $R e_{\tau}=100$ (red lines). LDV data are acquired one channel half-height downstream of the flush-mounted hot-film probe

of the wall shear stress fluctuations was due to heat loss to the substrate, which was largest in air and smallest in water. In the present investigation, a 60/40 per cent concentration by weight of glycerine/water was used as the working fluid, which had a thermal conductivity of $\lambda=0.38 \mathrm{~W} / \mathrm{mK}$ (Glycerine Producers' Assocation 1963). To ensure that the flush-mounted hot-film probe was able to capture the fluctuating wall shear stress correctly, an independent measure of the fluctuating wall shear stress was obtained by measuring the instantaneous streamwise velocity from within the viscous sublayer at $y / h<0.069$ with LDV, one channel half-height downstream of the flushmounted hot-film probe. Here, up to 150,000 data points were acquired with LDV from within the viscous sublayer at $R e_{\tau}=70,85$ and 100. Noting that the hot-film probe was sensitive to small drifts in ambient fluid temperature, Fig. 2, wall shear stress measurements with the hot-film probe were acquired over a duration of around $7 \mathrm{~h}$, where circa 5 million data points were collected at each Reynolds number. Here, the voltage output from the hot-film probe was linearly interpolated across two calibration curves separated by typically $1^{\circ} \mathrm{C}$ as shown in Fig. 2. Probability density functions (PDFs) of the fluctuating wall shear stresses acquired with LDV and the hot-film probe at $R e_{\tau}=70,85$ and 100 are shown in Fig. 5 . Excellent agreement can be seen across the two independent measurement techniques, illustrating that the hot-film probe correctly captures the wall shear stress.

\section{Conditional events}

The spatiotemporal low- and high-drag phenomena occur randomly in space and time in turbulent channel flow (Whalley et al. 2017; Kushwaha et al. 2017). Therefore to detect 
(a)

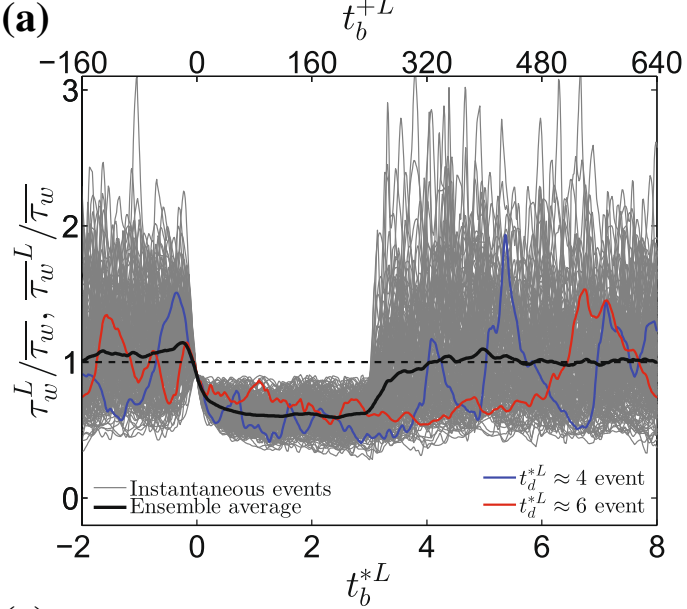

(c)

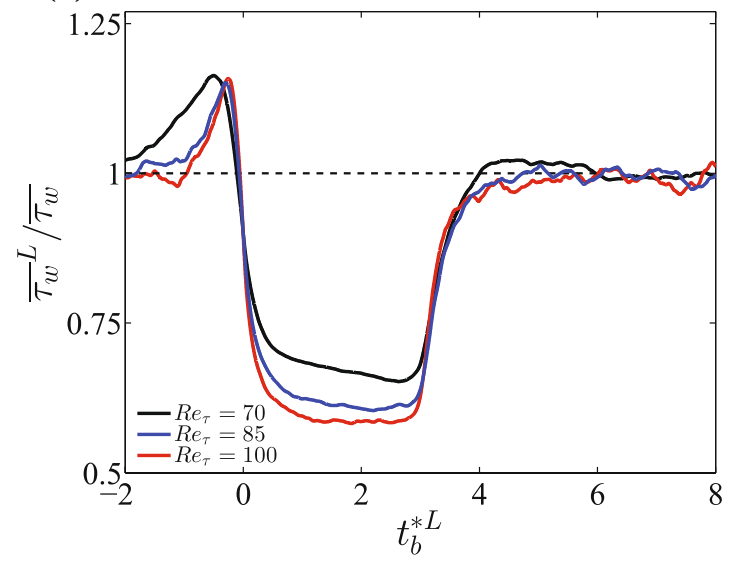

Fig. 6 a, b Example of instantaneous (201 events; grey thin lines) and ensemble-averaged (black thick line) wall shear stress during lowdrag events at $R e_{\tau}=85$. Shown are conditional samples at (a) the start of low-drag events and at (b) the end of low-drag events. The blue thick lines and red thick lines highlight an instantaneous lowdrag event with a duration of $t_{d}^{* L} \approx 4$ and $t_{d}^{* L} \approx 6$, respectively. The

these events experimentally, the wall shear stress is measured at a single point on the wall for long periods of time, typically hours. Whilst sampling the wall shear stress, the instantaneous streamwise velocity is captured simultaneously at discrete locations above the wall with LDV. Post-processing the wall shear stress signals with conditional sampling techniques provides 100 s of similar low- and high-drag events which are then ensemble averaged to reveal information on the average wall shear stress footprint and structure of the streamwise velocity during the low- and high-drag phenomena. Throughout the analyses presented, the low-drag events, or intervals of so-called "hibernating" turbulence, are conditionally sampled when the instantaneous wall shear stress falls $10 \%$ below the time-averaged wall shear stress for a duration of $t^{* L}=t^{L} \overline{u_{\tau}} / h>3$, or $t^{+L}=t^{L}{\overline{u_{\tau}}}^{2} / \nu>190$, 240 and 300 for $R e_{\tau}=70,85$ and 100, respectively, which is typically one order of magnitude larger than the integral

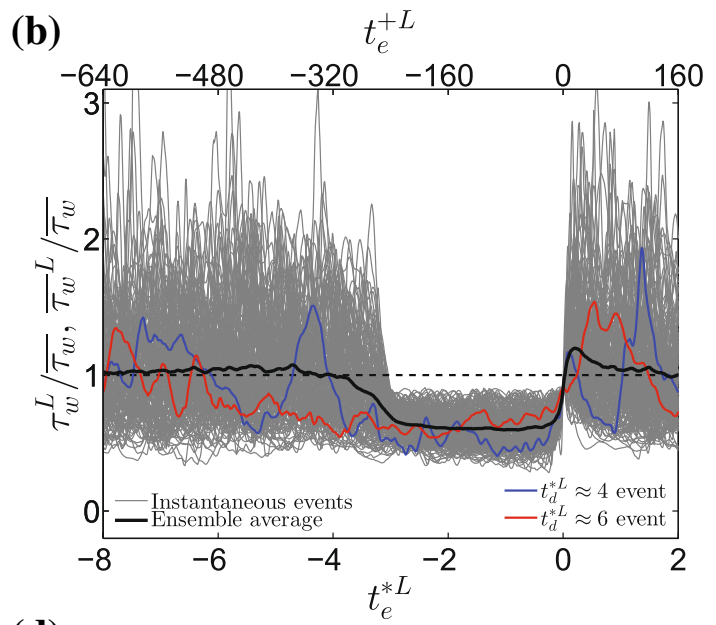

(d)

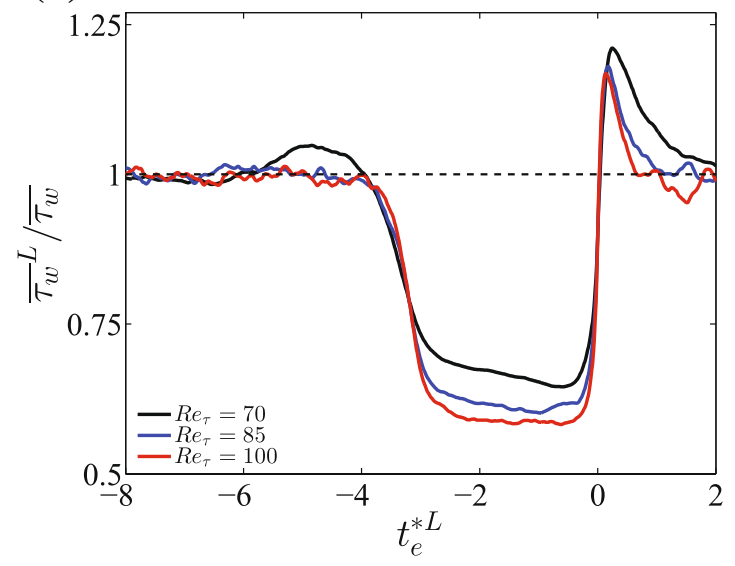

time-averaged wall shear stress is shown by the black dashed line. (c, d) Ensemble-averaged wall shear stress at $\mathbf{c}$ the start of low-drag events and at $\mathbf{d}$ the end of low-drag events at $R e_{\tau}=70$ (3003 events; black line), $R e_{\tau}=85$ (1017 events; blue line) and $R e_{\tau}=100$ (882 events; red line)

time scale of the flow. Similarly, the high-drag events are conditionally sampled when the instantaneous wall shear stress rises $5 \%$ above the time-averaged wall shear stress for a duration of $t^{* H}=t^{H} \overline{u_{\tau}} / h>2.5$ (or $t^{+H}>160,200$ and 250 for $R e_{\tau}=70,85$ and 100, respectively). Using highdrag criteria analogous to the low-drag criteria over the same absolute period of time (i.e. instantaneous wall shear stress rising $10 \%$ above the time-averaged wall shear stress for a duration of $t^{* H}>3$ ) yields very few high-drag events. Therefore the criteria for the high-drag phenomena are relaxed to ensure meaningful statistics. That said, sensitivity analyses on the conditional sampling criteria, for example, sampling on stress data which persists for $t^{* L, H}>2.5$ or $t^{* L, H}>3.5$, provides essentially the same results (Whalley et al. 2017; Kushwaha et al. 2017). Thus, our low- and high-drag results are rather insensitive to this precise choice of duration. 


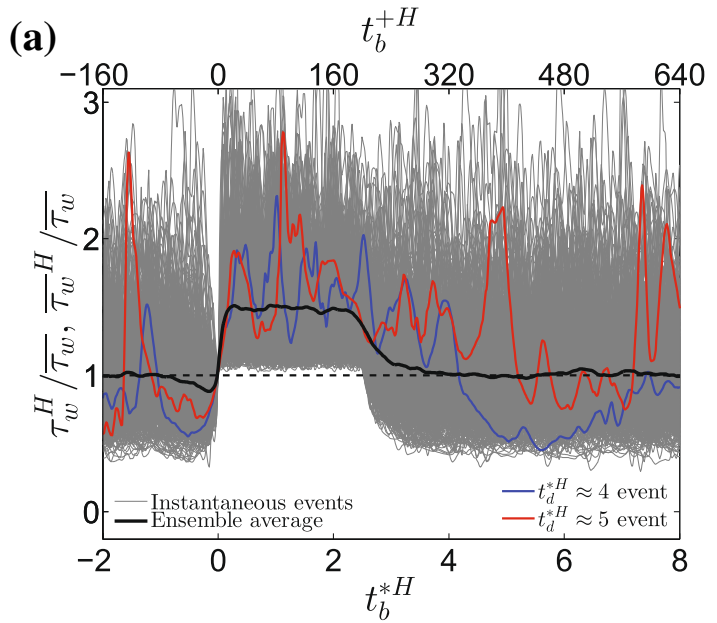

(c)

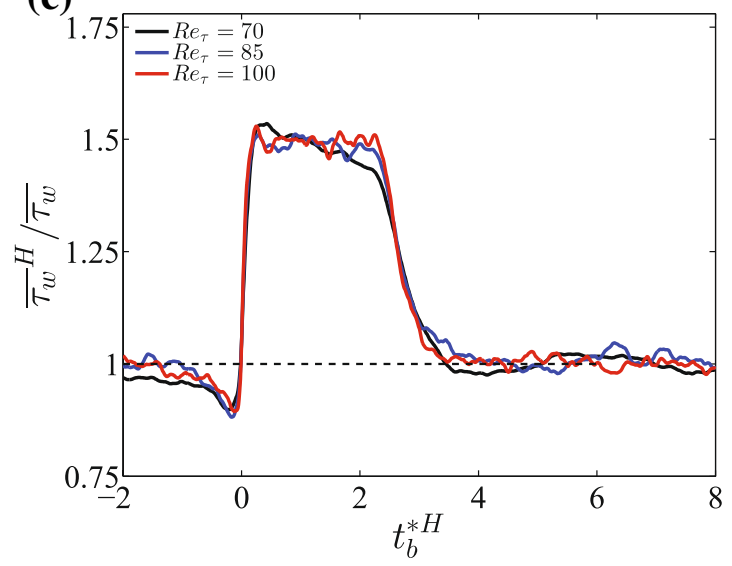

Fig. 7 a, b Example of instantaneous (475 events; grey thin lines) and ensemble-averaged (black thick line) wall shear stress during high-drag events at $R e_{\tau}=85$. Shown are conditional samples at a the start of high-drag events and at $\mathbf{b}$ the end of high-drag events. The blue thick lines and red thick lines highlight an instantaneous highdrag event with a duration of $t_{d}^{* H} \approx 4$ and $t_{d}^{* H} \approx 5$, respectively. The

Figures 6 and 7 show examples of instantaneous and ensemble-averaged wall shear stress during the low- and high-drag phenomena, respectively. The data are normalized by the time-averaged wall shear stress of the entire data set, $\overline{\tau_{w}}$. The grey thin lines show hundreds of instantaneous wall shear stress events at $R e_{\tau}=85$. In Figs. 6a and $7 \mathrm{a}$ each low- and high-drag event is shifted to begin at $t_{b}^{* L, H}=0$ to show how the start of the phenomena evolves with time. Here, the subscript $b$ notes the beginning of the low- or high-drag phenomena with $t_{b}^{* L, H}=0$ corresponding to the time when the instantaneous wall shear stress initially falls to $10 \%$ below or rises to $5 \%$ above the timeaveraged wall shear stress, respectively. The black thick line shows the corresponding ensemble average through all the instantaneous events. The blue thick lines show instantaneous events with durations of $t_{d}^{* L, H} \approx 4$, and the thick red line shows an instantaneous low-drag event with
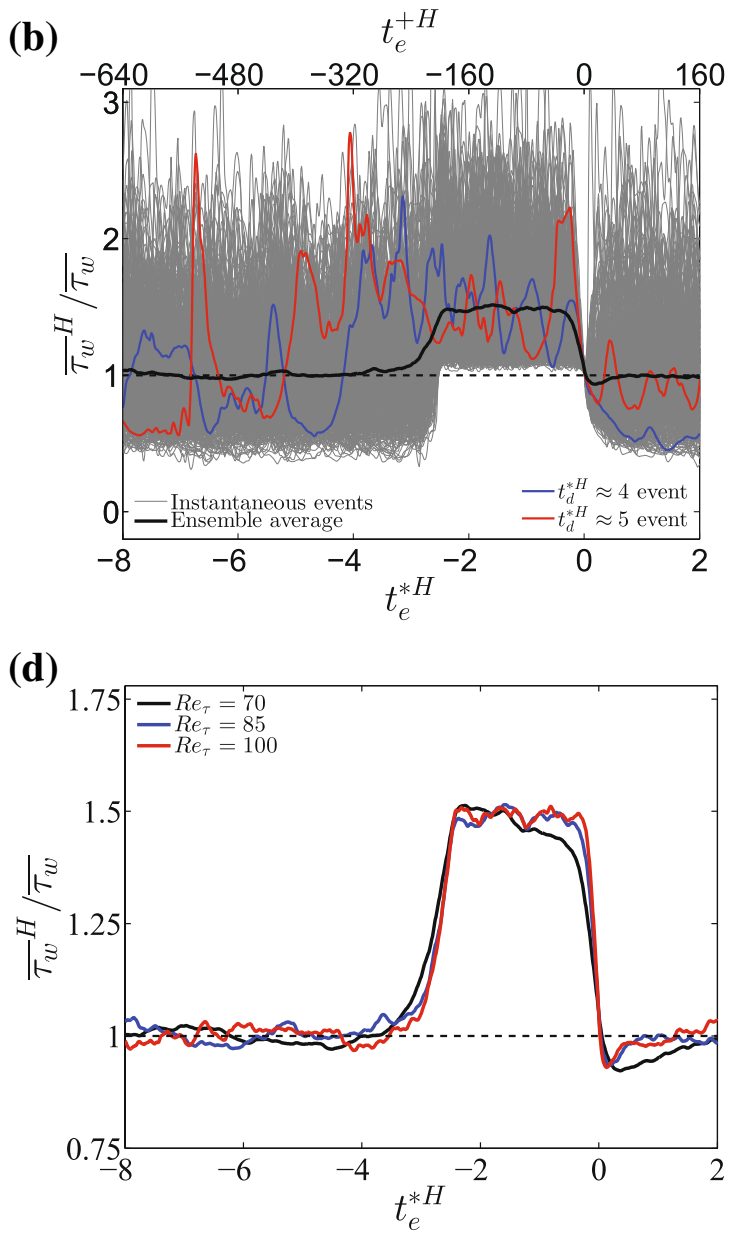

time-averaged wall shear stress is shown by the black dashed line. c, d Ensemble-averaged wall shear stress at (c) the start of highdrag events and at (d) the end of high-drag events at $R e_{\tau}=70$ (1422 events; black line), $R e_{\tau}=85$ (475 events; blue line), $R e_{\tau}=100$ (399 events; red line)

a duration of $t_{d}^{* L} \approx 6$ or an instantaneous high-drag event with a duration of $t_{d}^{* H} \approx 5$. Whilst events with durations of $t_{d}^{* L, H}>6$ are detected, they occur infrequently. Generally, the frequency of longer lasting events reduces with increasing Reynolds number (Whalley et al. 2017). Figures $6 \mathrm{~b}$ and $7 \mathrm{~b}$ show the same low- and high-drag events from Figs. 6a and 7a, but shifted in time to show how the end of the low- and high-drag phenomena evolves with time. Here, the subscript $e$ notes the end of the low- or high-drag phenomena with $t_{e}^{* L, H}=0$ corresponding to the time when the instantaneous wall shear stress is $10 \%$ below or $5 \%$ above the time-averaged wall shear stress on exit from either the low- or high-drag trajectory, respectively. The duration of events scaled in viscous time units (e.g. $\left.t^{+L, H}\right)$ is shown on the upper time axes as an alternative time criterion to detect low- and high-drag events. Under a 

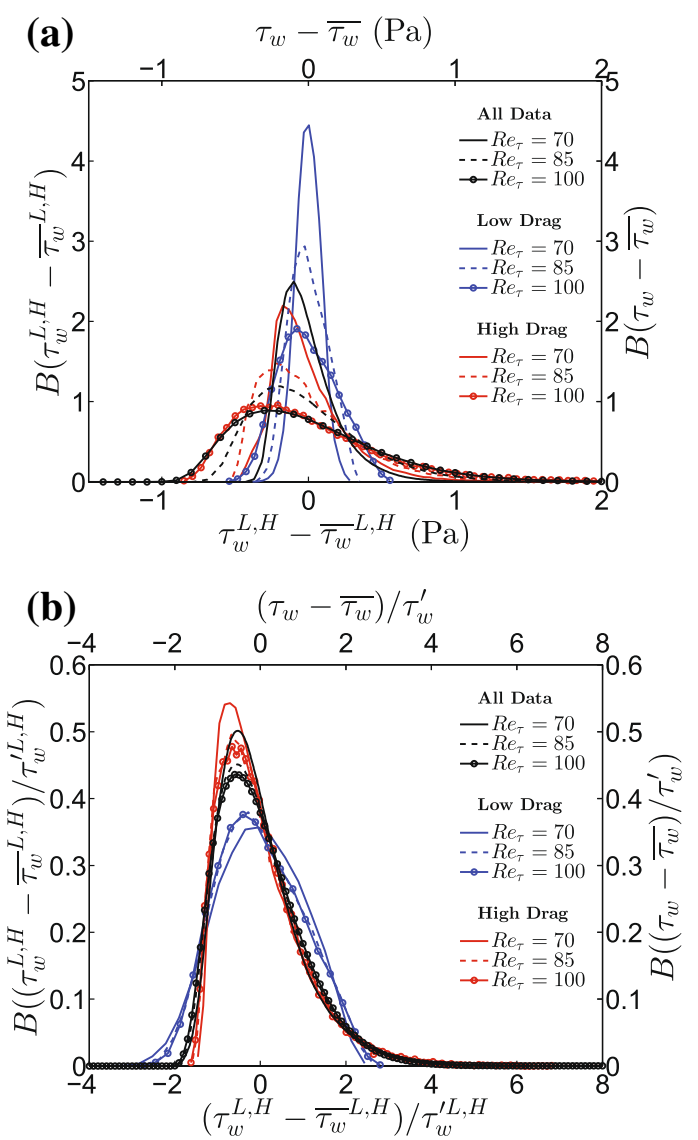

(c)

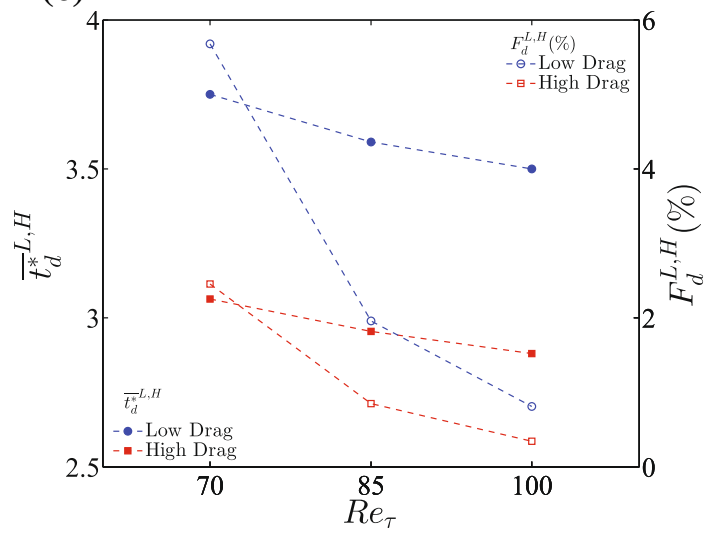

Fig. 8 a PDFs of the fluctuating wall shear stress during low-drag events (blue lines), high-drag events (red lines) and entire data series (black lines) at $R e_{\tau}=70$ (solid lines), $R e_{\tau}=85$ (dashed lines) and $R e_{\tau}=100$ (solid lines with symbol). b PDFs of normalized fluctuating wall shear stress during low- and high-drag events, and entire data series. Line colour the same as in a. c Average duration of low-drag events (filled blue circle) and high-drag events (filled red square), and the fraction of time spent in low-drag events (open blue circle) and high-drag events (open red square) as functions of Reynolds number

fixed time duration of $t^{*}>3$, the duration of events measured in viscous time units increases with friction Reynolds number.
The ensemble averages through all the conditionally sampled wall shear stress data, at the start and end of the lowand high-drag phenomena, for $R e_{\tau}=70,85$ and 100, are shown in Figs. 6c, d and 7c, d. From an averaged point of view, the low-drag phenomena start and end with a higher than average peak in wall shear stress which persists for a duration of $t_{d}^{* L} \approx 2$ at $R e_{\tau}=70$, and $t_{d}^{* L} \approx 1$ at $\operatorname{Re}_{\tau}=85$ and 100. In between the two high wall shear stress peaks, the low-drag phenomena plateau to an almost constant value during $0.7<t^{* L}<2.8$, at around $40 \%$ below the time-averaged wall shear stress, with some small Reynolds number dependence. The high-drag phenomena appear as an almost mirror image of the low-drag phenomena about the timeaveraged wall shear stress: the ensemble-averaged high-drag events start and end with a lower than average peak in wallshear stress which rises to an almost constant value of wall shear stress during $0.7<t^{* H}<2.3$, at around $50 \%$ above the time-averaged wall shear stress, across all Reynolds numbers tested. A subtle difference is the relative magnitudes of the ensemble-averaged wall shear stresses during the two plateau regions.

PDFs of the fluctuating wall shear stresses of the low-drag, high-drag and entire data series are shown in Fig. 8a. The distributions of the entire data (black lines) and high-drag data (red lines) are positively skewed, which is in contrast to the distributions of the low-drag data (blue lines) which are more symmetric. Normalizing the data sets by the corresponding wall shear stress r.m.s collapses the data at $R e_{\tau}=85$ and 100, Fig. 8b. Interestingly, Kushwaha et al. (2017) observed that the near-wall region of channel flow was dominated by large-scale stripy structures which were orientated at an oblique angle of $20^{\circ}-30^{\circ}$ to the mean flow at $R e_{\tau}=70$, an observation which is in excellent agreement with previous numerical and experimental investigations (Hashimoto et al. 2009; Tsukahara et al. 2010), and is indicative of the later stages of transition to turbulence. These observations may explain the lack of collapse of data at $R e_{\tau}=70$, including the subtle Reynolds number dependence in the ensemble-averaged low wall shear stress data; Fig $6 \mathrm{c}, \mathrm{d}$.

The average duration $\left(\overline{t_{d}^{*}}\right)$ and the fraction of the time spent $\left(F_{d}\right)$ by the flow in a low- or high-drag state is shown in Fig. 8c,

${\overline{t_{d}^{*}}}^{L, H}=\frac{\sum_{n=1}^{N} t_{d, n}^{* L, H}}{N}$

$F_{d}^{L, H}=\frac{\sum_{n=1}^{N} t_{d, n}^{* L, H}}{T^{*}}$

Here, $t_{d, n}^{* L, H}$ is the duration of the $n$th low- or high-drag interval, $N$ is the total number of low- or high-drag intervals, and $T^{*}$ is the total signal duration. The absolute values of these 
(a)

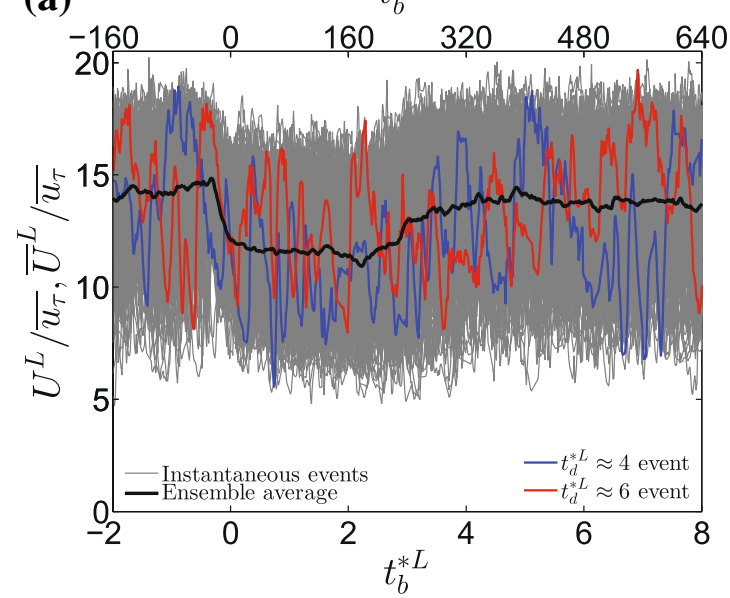

(c)

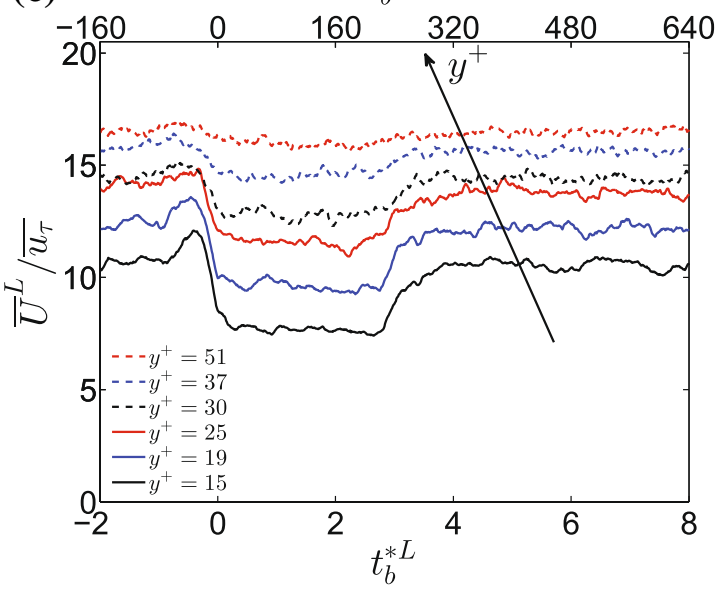

Fig. 9 a, b Example of instantaneous (201 events; grey thin lines) and ensemble-averaged (black thick line) streamwise velocity scaled with time-averaged wall shear stress at $y^{+}=15$ during low-drag events at $R e_{\tau}=85$. Shown are conditional samples at (a) the start of lowdrag events and at (b) the end of low-drag events. The blue thick lines and red thick lines highlight an instantaneous low-drag event with a

quantities are dependent on the choice of conditional sampling criteria; however, the Reynolds number dependence is indicative, irrespective of the criteria used. The average durations of the low- and high-drag events remains almost constant at a value slightly larger than the chosen duration criterion as the Reynolds number increases from $R e_{\tau}=70$ to 100 . The fraction of time spent by the flow in low- and high-drag states decreases with increasing Reynolds number. Whilst the fraction of time spent in a low- or high-drag state is small, sub $1 \%$ at $R e_{\tau}=100$, it remains finite. The flow is intrinsically more likely to enter a state of low drag than high drag using our criteria, in accordance with DNS (Kushwaha et al. 2017).

Conditionally sampled and ensemble-averaged streamwise velocities during the low- and high-drag states at $R e_{\tau}=85$ are shown in Figs. 9 and 10, respectively. The
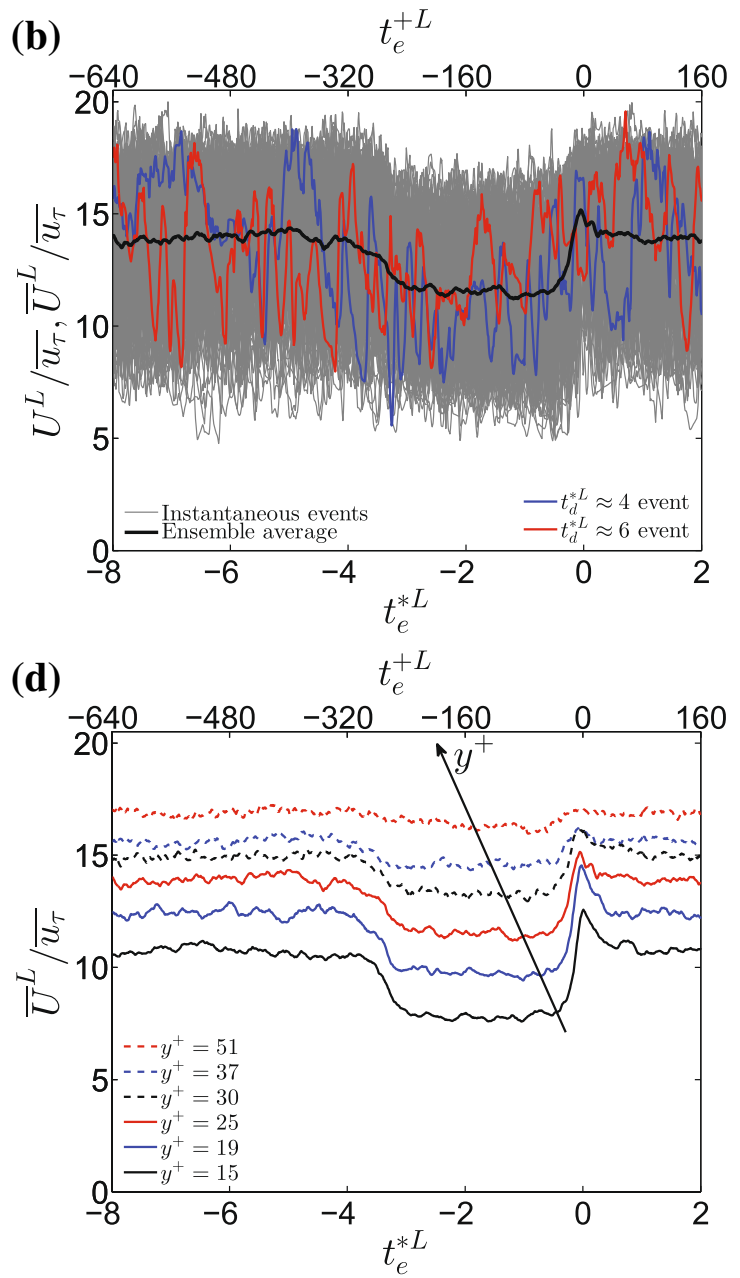

duration of $t_{d}^{* L} \approx 4$ and $t_{d}^{* L} \approx 6$, respectively. c, d Ensemble-averaged streamwise velocity at $R e_{\tau}=85$ at (c) the start of low-drag events and at (d) the end of low-drag events at $y^{+}=15$ (201 events; black solid line), $y^{+}=19$ (168 events; blue solid line), $y^{+}=25$ (175 events; red solid line), $y^{+}=30$ (155 events; black dashed line), $y^{+}=37$ (177 events; blue dashed line) and $y^{+}=51$ (141 events; red dashed line)

grey thin lines show instantaneous streamwise velocity aligned to accentuate the evolution of streamwise velocity at the beginning of the low-drag intervals, Fig. 9a, and at the end of the low-drag intervals, Fig. 9b. The thick black line shows an ensemble average through all the instantaneous conditionally sampled data, the thick blue line shows an instantaneous event with a duration of $t_{d}^{* L, H} \approx 4$, and the thick red line shows an instantaneous low-drag event with a duration of $t_{d}^{* L} \approx 6$. A distinct difference is observed in the instantaneous evolution of the streamwise velocity and wall shear stress signals. It was a condition when post-processing the wall shear stress data that the signal remained below or above a threshold for a given duration. Yet, conditionally sampling the streamwise velocity on the wall shear stress data shows that instantaneous streamwise velocities fluctuate largely around the ensemble-averaged data: see the blue 


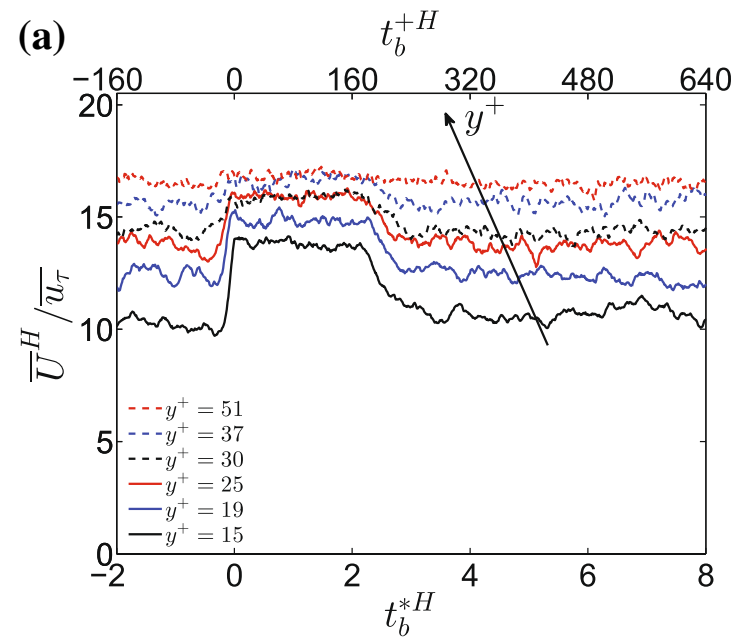

Fig. 10 a, b Ensemble-averaged streamwise velocity at $R e_{\tau}=85$ at (a) the start of high-drag events and at (b) the end of high-drag events at $y^{+}=15$ (100 events; black solid line), $y^{+}=19$ (81 events; blue

and red lines in Fig. 9a, b. However, close to the wall, on an ensemble-averaged point of view, the streamwise velocity mimics the wall shear stress signatures during the low- and high-drag states. There are small peaks or troughs at the start and end of the ensemble-averaged low- and high-drag intervals, respectively, joined by a plateau of almost constant streamwise velocity. The coherence between the wall shear stress and streamwise velocity is lost further away from the wall: see Figs. 9c, $d$ and 10a, b, which show ensembleaveraged data at various wall-normal distances. By $y^{+} \approx 50$, the conditionally sampled and ensemble-averaged velocities appear constant, perhaps indicating a loss of communication with the wall.

Shown in Fig. 11a is the ensemble-averaged streamwise velocity plotted against wall-normal distance. The Prandtl-von Kármán log-law is shown by the blue dotted line. The blue closed symbols show the ensemble-averaged streamwise velocity measured during low-drag events, or intervals of hibernating turbulence. These data are averaged over the plateau region $t^{* L}=0.7-2.8$ and are scaled with the ensemble-averaged wall shear stress determined during the same time interval for LDV and the instantaneous wall shear stress for SPIV. The uncertainty bars for the LDV data represent the spread of streamwise velocity data within each conditionally sampled ensemble average. For further comparison, a recent family of TW solutions (Park and Graham 2015) at $R e_{\tau}=85$ are shown in Fig. 11a. Here, the upper branch solution (black dotted-dashed line) approaches the Prandtl-von Kármán log-law, whilst the lower branch solution (black dashed line) falls close to the low-drag data up to $y^{+} \approx 40$. Beyond $y^{+} \approx 40$, the low-drag data follows a gradient similar to the Prandtl-von Kármán log-law, as observed with DNS (Kushwaha et al. 2017). A sensitivity analysis

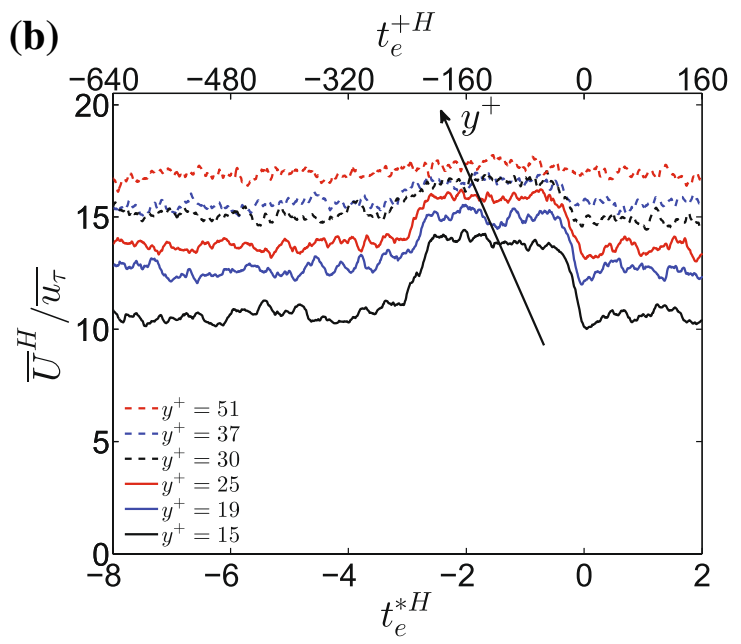

solid line), $y^{+}=25$ (84 events; red solid line), $y^{+}=30$ (79 events; black dashed line), $y^{+}=37$ (67 events; blue dashed line) and $y^{+}=51$ (65 events; red dashed line)

(pink open symbols) on the conditional sampling criteria at $R e_{\tau}=70$ shows that the conditionally averaged streamwise velocity data is insensitive to small changes in the time criterion used to detect hibernating turbulence. Here, the conditionally sampled streamwise velocity data collapses when using the criteria that hibernating turbulence occurs once the instantaneous wall shear stress drops to $10 \%$ below the mean wall shear stress for a duration of $t^{* L}>2.7,3$ or 3.3. Making the wall shear stress criterion more stringent, by conditionally sampling data once the instantaneous wall shear stress drops to $75 \%, 80 \%$ or $85 \%$ below the mean wall shear stress, causes a small shift in the conditionally sampled streamwise velocity data as shown in Fig. 11a; these same trends have been observed in a recent DNS study (Kushwaha et al. 2017). We note that Hutchins et al. (2011) conditionally sampled streamwise velocity on low-drag events in a high Reynolds number turbulent boundary layer flow. They used the criterion that the flow enters into a state of low drag when the skin friction fluctuation was below zero and observed an upward shift in the log-law rather than a change in log-law gradient. This could be a consequence of Reynolds number as their study was conducted at $R e_{\tau}=14200$, geometry, or that their conditional sampling technique did not have a time criterion which may have caused the averaging out of the rare low-drag events observed here. Also shown in Fig. 11a is the high-drag data (red closed symbols), ensemble-averaged over the plateau region $t^{* H}=0.7-2.3$ and scaled with the ensemble-averaged high wall shear stress data accordingly. Generally, these data show Reynolds number dependence, falling further below the classical log-law with increasing Reynolds number, an observation which is also seen with DNS (Kushwaha et al. 2017) and in much 

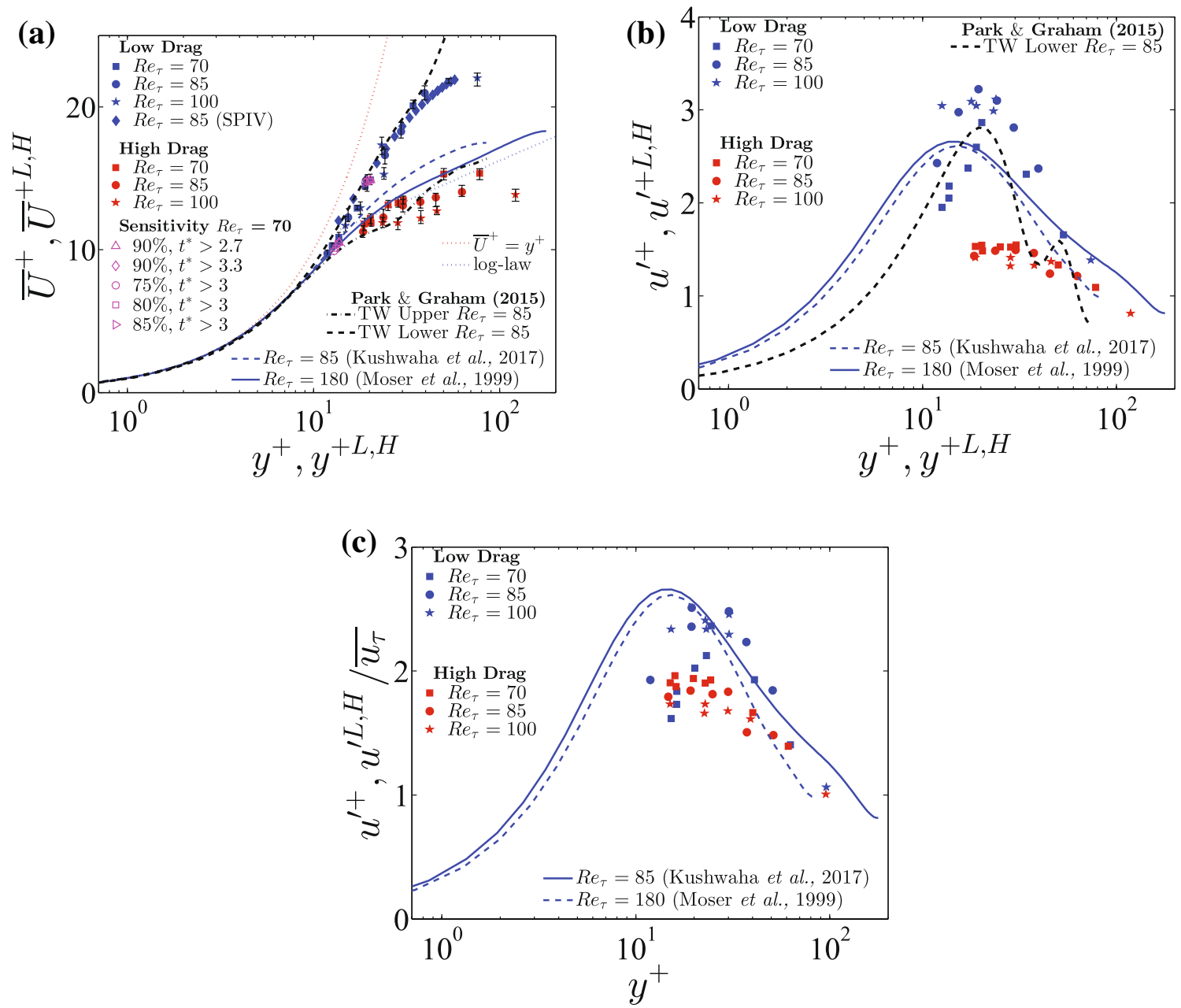

Fig. 11 a Conditionally averaged streamwise velocity (low-drag events-closed blue symbols; high-drag events-closed red symbols) scaled with conditionally averaged wall shear stress: $R e_{\tau}=70$ (filled blue square, filled red square), $R e_{\tau}=85$ (filled blue circle, filled red circle), $R e_{\tau}=100$ (filled blue star, filled red star) and SPIV at $R e_{\tau}=85$ (filled blue diamond). A sensitivity analysis on the conditional sampling criteria at $R e_{\tau}=70$ shows conditionally averaged streamwise velocity data when using the criteria that the flow enters into a state of hibernation once the instantaneous wall shear stress drops $10 \%$ below the mean wall-shear stress for a duration of $t^{* L}>$ 2.7 (open pink triangle) and $t^{* L}>3.3$ (open pink diamond), and also when the instantaneous wall shear stress drops $75 \%$ (open pink cir-

higher Reynolds number turbulent boundary layer flows (Hutchins et al. 2011).

The turbulence intensity of the low- and high-drag events are shown in Fig. 11b. Generally, the low- and high-drag data sit above and below the canonical DNS data, respectively, which is largely due to scaling with the corresponding ensemble-averaged low- and high-wall shear stress. Both the low- and high-drag data sets exhibit a peak in intensity around $y^{+L, H} \approx 20$. The lower branch TW solution captures the qualitative trend of the low-drag data over $10<y^{+}<40$. Scaling with the time-averaged wall shear stress, Fig. 11c, cle), $80 \%$ (open pink square) and $85 \%$ (right facing pink triangle) below the mean wall shear stress for a duration of $t^{* L}>3$. The red dotted line is the viscous sublayer profile: $\bar{U}^{+}=y^{+}$, blue dotted line is the Prandtl-von Kármán log-law: $\bar{U}^{+}=2.44 \ln y^{+}+5$. Nonlinear TW solutions from Park and Graham (2015) at $R e_{\tau}=85$ : lower branch (black dashed line) and upper branch (black dotted-dashed line). The blue dashed line is DNS data at $R e_{\tau}=85$ (Kushwaha et al. 2017) and the blue solid line is DNS data at $R e_{\tau}=180$ (Moser et al. 1999). b Conditionally averaged streamwise turbulence intensity scaled with conditionally averaged wall shear stress. c Conditionally averaged streamwise turbulence intensity data from (b) scaled with time-averaged friction velocity. Symbols for part $(\mathbf{b}, \mathbf{c})$ are the same as in $\mathbf{a}$

generally shows that the low-drag data has a higher turbulence intensity across the majority of the channel half-height when compared with the high-drag intensity data. Strong spatiotemporal intermittencies have been observed within transitional wall-turbulent flows, even close to locations where the fluid flow is relatively quiescent, containing little drag. Hof et al. (2006) showed that spatiotemporal intermittencies can have long lifetimes, remaining within the flow throughout the transition process and into the fully turbulent flow regime (Avila et al. 2010). The large levels of streamwise turbulence intensity observed in the low-drag intervals 

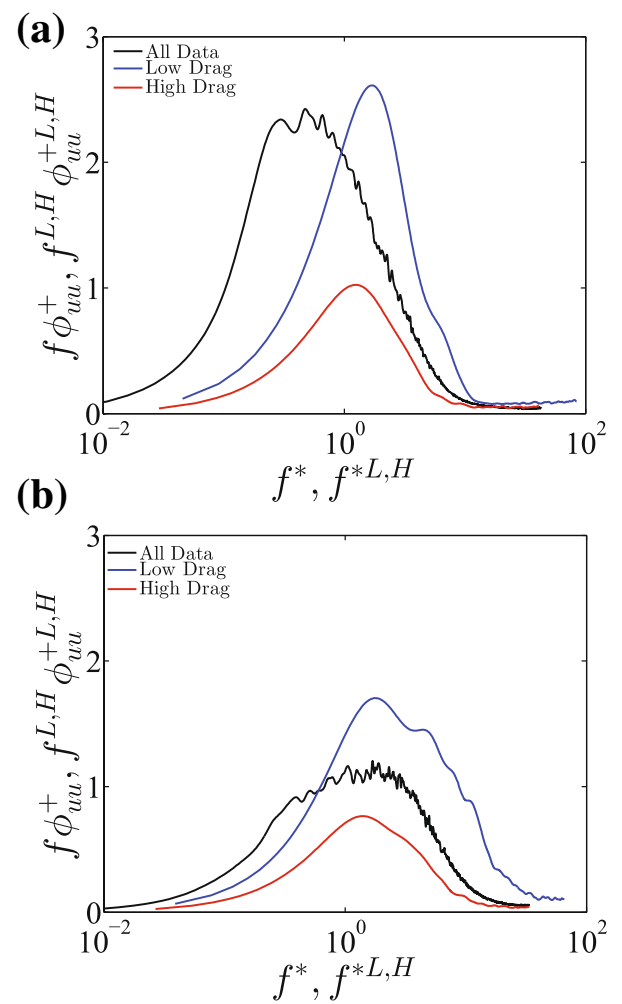

Fig. 12 Pre-multiplied energy spectra of streamwise velocity at (a) $y^{+}=y^{+L, H}=19\left(R e_{\tau}=70\right)$ and (b) $y^{+}=y^{+L, H}=40\left(R e_{\tau}=85\right)$. The black solid line shows data from the entire data series, the blue solid line shows low-drag data and the red solid line shows high-drag data. The entire data series is normalized by the time-averaged friction velocity, and the low- and high-drag data are normalized by the conditionally averaged low- and high-friction velocities, respectively

may be a reflection of the strong spatiotemporal intermittency observed generally in the transition region.

Figure 12 shows pre-multiplied energy spectra of streamwise velocity, $f \phi_{u u}^{+}$versus frequency, $f^{*}=1 / t^{*}$, where $\int_{\infty}^{-\infty} f \phi_{u u}^{+} d(\log f)=u^{\prime+2}$. Figure 12 a shows data at $y^{+}=y^{+L, H}=19\left(R e_{\tau}=70\right)$, and Fig 12b shows data at $y^{+}=y^{+L, H}=40\left(\operatorname{Re}_{\tau}=85\right)$. Comparison across the two figures shows that the low- and high-drag data have peaks in energy at $f^{* L} \approx 1.7$ and $f^{* H} \approx 1.3$. The energy distribution of the low-drag data is shifted to larger frequencies at $f^{* L} \gtrsim 1$. The high-drag energy spectrum is encapsulated within the low-drag energy spectrum.

To elucidate on the distributions of the streamwise velocity during the low- and high-drag events, PDFs of the instantaneous streamwise velocity scaled with the instantaneous wall shear stress are shown in Fig 13a, b. Figure 13a shows data at $y^{+}=y^{+L, H}=19\left(\operatorname{Re}_{\tau}=70\right)$ and Fig. 13b shows data at $y^{+}=y^{+L, H}=40\left(\operatorname{Re}_{\tau}=85\right)$. Plotting the entire data series (black solid lines) at these two locations shows, from an instantaneous point of view, that the flow spends some portion of time above and below the MDR
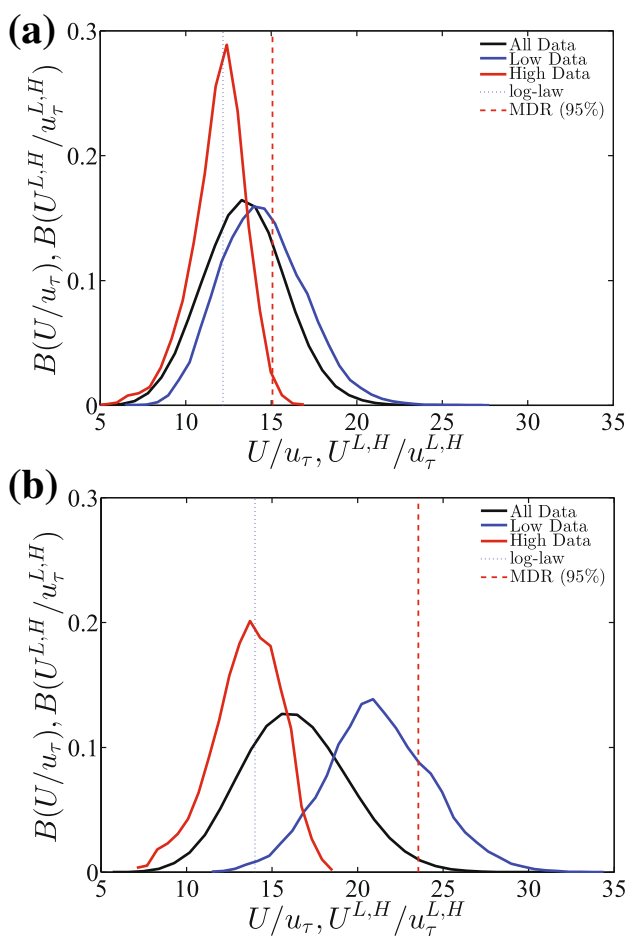

Fig. 13 a, b PDFs of conditionally sampled instantaneous streamwise velocity scaled with instantaneous wall shear stress during low-drag events (blue solid line) and high-drag events (red solid line), alongside PDFs of all data (black solid line) scaled with instantaneous wall shear stress at a $y^{+}=y^{+L, H}=19, R e_{\tau}=70$, b $y^{+}=y^{+L, H}=40$, $R e_{\tau}=85$. The blue dotted line shows the location of the Prandtl-von Kármán log-law: $\bar{U}^{+}=2.44 \ln y^{+}+5$, and the red dashed line shows the location of the $95 \%$ confidence interval of the MDR asymptote: $\bar{U}^{+}=11.4 \ln y^{+}-18.5$ (Virk et al. 1970; Graham 2014)

asymptote and classical log-law, less so beyond the MDR asymptote with increasing distance from the wall. Conditionally sampling the entire data series to separate the low-drag data (blue solid lines) shows that during intervals of hibernating turbulence, the fluid flow spends some part of its trajectory around and below the Prandtl-von Kármán log-law as well as significantly beyond the MDR asymptote. In contrast, by $y^{+H}=40$, the high-drag data (red solid lines) remains central to the classical log-law.

A typical instantaneous snapshot of the streamwise velocity during a low-drag event, captured with SPIV at $R e_{\tau}=85$, is shown in Fig. 14a. At times during the lowdrag event, a counter-rotating vortex pair meanders in the spanwise direction lifting low-speed fluid from the nearwall region towards the centre of the channel, similar to an ejection event. As the turbulent flow structure meanders in the spanwise direction, it is likely that the spanwise location of minimum wall shear stress is not directly above the location of the wall shear stress probe at $z^{+}=0$, but is instead located at some small distance on either side 

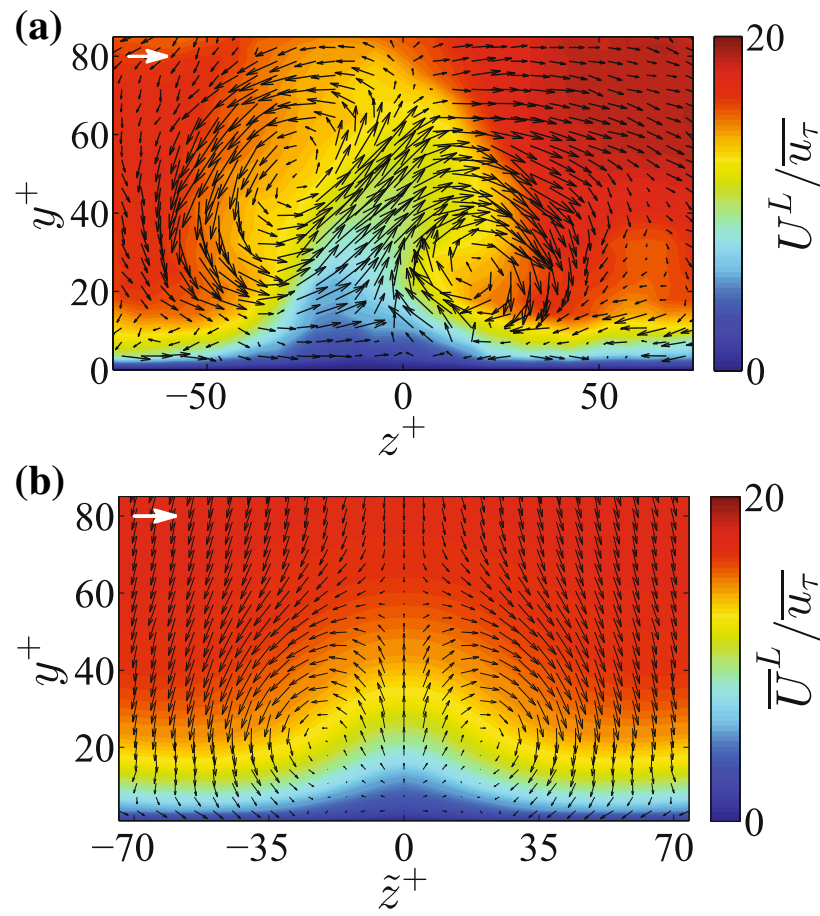

Fig. 14 a Instantaneous and $\mathbf{b}$ ensemble-averaged streamwise velocity scaled with time-averaged wall shear stress at $R e_{\tau}=85$ during low-drag events captured using SPIV. The ensemble-average comprises 207 SPIV snapshots acquired over four low-drag events. As part of the ensemble averaging the data are symmetrized about $\tilde{z}^{+}=0$. Black arrows show wall-normal and spanwise velocities. The $\left(y^{+}, z^{+}\right)$axes are scaled with the time-averaged wall shear stress. For reference, the white arrow in a has length $2 u_{\tau}$ and in $\mathbf{b}$ has length $0.5 u_{\tau}$

of the probe. To take this effect into account during the ensemble-averaging process, the spanwise location of minimum streamwise velocity at $y^{+}=11$ over $-15<z^{+}<15$ is used as an indicative measure for the true spanwise location of minimum wall shear stress, $\tilde{z}^{+}=0$. The ensemble-averaged streamwise velocity throughout the four low-drag events captured with SPIV is shown in Fig. 14b, symmetrized around $\tilde{z}^{+}=0$. Here, half of the ensembleaveraged velocity field is mirrored around $\tilde{z}^{+}=0$, then the two halves are averaged. From an averaged point of view, the hibernating turbulence flow structure is characterized by a low-stress streak which is flanked on either side by a quasi-streamwise vortex; this is in excellent agreement with recent DNS (Whalley et al. 2017; Kushwaha et al. 2017).

\section{Conclusions}

This paper presents an experimental investigation into spatiotemporal intermittencies in turbulent channel flows of a Newtonian fluid at friction Reynolds numbers of $R e_{\tau}=70$,
85 and 100, close to transition to turbulence. Low- and highdrag states have been identified by measuring the wall shear stress at a single point on the wall, whilst, simultaneously, velocity data have been acquired with either LDV or SPIV. Post-processing the data with conditional sampling techniques has provided information on the instantaneous and ensemble-averaged turbulent flow structure during the lowand high-drag intervals.

The ensemble-averaged low-drag events, or intervals of so-called hibernating turbulence, show a streamwise velocity profile which falls close to the lower branch of a recently discovered non-linear TW solution to the Navier-Stokes equations. In contrast, the ensemble-averaged high-drag events show streamwise velocity profiles which fall further below the classical log-law with increasing Reynolds number. The instantaneous streamwise velocity signatures are markedly different during the intervals of low and high drag. Comparatively higher and lower turbulence intensity is seen up to $y^{+} \approx 60$ for the low- and high-drag intervals, respectively.

The ensemble-averaged low wall shear stress time signatures appear symmetric, characterized by a higher than average peak in stress at the start and end of the low-drag trajectory, bridged in between by an almost constant value of wall shear stress. The low-drag events are characterized by a low-stress streak which is flanked on either side by a streamwise vortex, forming a counter-rotating vortex pair. A similar flow structure has recently been observed with the aforementioned lower branch TW solution (Whalley et al. 2017). The high wall shear stress time signatures are an almost mirror image of the low-stress data about the timeaveraged wall shear stress. Below $y^{+} \approx 40$, there is a strong correlation between the wall shear stress and streamwise velocity data, with the ensemble-averaged streamwise velocity data mimicking the wall shear stress signatures. Beyond $y^{+} \approx 40$, there is little correlation, perhaps suggesting a lack of communication with the wall. PDFs of the wall shear stress data show good collapse when scaled with the wall shear stress r.m.s. There is some noticeable Reynolds number dependence at $R e_{\tau}=70$, as observed in the ensembleaveraged wall shear stress signatures, and may be due to the near-wall stripy structure observed in channel flows at the later stages of transition to turbulence (Hashimoto et al. 2009; Tsukahara et al. 2010).

The excursions into low- and high-drag trajectories is rare in Newtonian fluid flow based on the current choice of conditional sampling criteria. Intrinsically, the fluid flow is more likely to enter a state of low drag than high drag with the fraction of time spent in either state being a function of Reynolds number. Although the time spent in either trajectory is small, sub 5\%, it remains finite at $R e_{\tau}=100$.

Acknowledgements This work has been supported by the Engineering and Physical Sciences Research Council (EPSRC) under grant number 
EP/J018163/1. We thank the EPSRC for the loan of the SPIV system used to carry out part of this research. We would like to acknowledge Mr. Rishav Agrawal for his assistance in determining the critical Reynolds number of the channel flow facility.

Open Access This article is distributed under the terms of the Creative Commons Attribution 4.0 International License (http://creativeco mmons.org/licenses/by/4.0/), which permits unrestricted use, distribution, and reproduction in any medium, provided you give appropriate credit to the original author(s) and the source, provide a link to the Creative Commons license, and indicate if changes were made.

\section{References}

Adrian R (2007) Hairpin vortex organisation in wall turbulence. Phys. Fluids 19(041):301

Alavyoon F, Hennigson DS, Alfredsson PH (1986) Turbulent spots in plane poiseuille flow-flow visualization. Phys. Fluids 29:1328-1331

Alfredsson PH, Johansson AV, Haritonidid JH, Eckelmann H (1988) The fluctuating wall-shear stress and the velocity field in the viscous sublayer. Phys Fluids 31:1026-1033

Avila M, Willis AP, Hof B (2010) On the transient nature of localized pipe flow turbulence. J Fluid Mech 646:127-136

Choi KS (1989) Near-wall structure of a turbulent boundary layer with riblets. J Fluid Mech 208:417-458

Choi KS, Jukes TN, Whalley RD (2011) Turbulent boundarylayer control with plasma actuators. Philos Trans R Soc A 369:1443-1458

Christensen KT (2004) The influence of peak-locking errors on turbulence statistics computed from PIV ensembles. Exp Fluids 36:484-497

Dean RB (1978) Reynolds number dependence of skin friction and other bulk flow variables in two-dimensional rectangular duct flow. J Fluids Eng 100:215-223

Eckhardt B, Schnieder TM, Hof B, Westerweel J (2007) Turbulence transition in pipe flow. Ann Rev Fluid Mech 39:447-468

Eckhardt B, Faisst H, Schmiegel A, Schneider TM (2008) Dynamical systems and the transition to turbulence in linearly stable shear flows. Philos Trans R Soc A 366:1297-1315

Garcia-Mayoral R, Jimenez J (2011) Drag reduction by riblets. Philos Trans R Soc A 369:1412-1427

Gibson JF, Halcrow J, Cvitanovic P (2008) Visualizing the geometry of state space in plane couette flow. J Fluid Mech 611:107-130

Glycerine Producers' Assocation (1963) Physical properties of glycerine and its solutions. Glycerine Producers' Association, New York

Graham MD (2014) Drag reduction and the dynamics of turbulence in simple and complex fluids. Phys Fluids 26(101):301

Hashimoto S, Hasobe A, Tsukahara T, Kawaguchi Y, Kawamura H (2009) An experimental study on turbulent-stripe structure in transitional channel flow. In: Sixth international symposium on turbulence, heat and mass transfer, Begell House

Head MR, Bandyopadhyay P (1981) New aspects of turbulent boundary layer structure. J Fluid Mech 107:297-338

Hof B, van Doorne CWH, Westerweel J, Nieuwstadt FTM, Faisst H, Eckhardt B, Wedin H, Kerswell RR, Waleffe F (2004) Experimental observations of nonlinear traveling waves in turbulent pipe flow. Science 305(5690):1594-1598

Hof B, Westerweel J, Schneider TM, Eckhardt B (2006) Finite lifetime of turbulence in shear flows. Nature 443:59-62

Hof B, de Lozar A, Avila M, Tu X, Schnieder TM (2010) Eliminating turbulence in spatially intermittent flows. Science 327:1491-1494
Hutchins N, Choi KS (2002) Accurate measurements of local skin friction coefficient using hot-wire anemometry. Prog Aerosp Sci 38:421-446

Hutchins N, Hambleton WT, Marusic I (2005) Inclined cross-stream stereo particle image velocimetry measurements in turbulent boundary layers. J Fluid Mech 541:21-54

Hutchins N, Monty JP, Ganapathisubramani B, Ng HCH, Marusic I (2011) Three-dimensional conditional structure of a high-Reynolds-number turbulent boundary layer. J Fluid Mech 673:255-285

Jimenez J, Moin P (1991) The minimal flow unit in near-wall turbulence. J Fluid Mech 225:213-240

Karniadakis GE, Choi KS (2003) Mechanisms on transverse motions in turbulent wall flows. Ann Rev Fluid Mech 35:45-62

Kawahara G, Uhlmann M, van Veen L (2012) The significance of simple invariant solutions in turbulent flows. Ann Rev Fluid Mech 44:203-225

Kerswell RR (2005) Recent progress in understanding the transition in pipe flow. Nonlinearity 18(6):R17-R44

Kline SJ, McClintock FA (1953) Describing uncertainties in single sample experiments. Mech Eng 75:3-8

Kline SJ, Reynolds WC, Schraub FA, Runstadler PW (1967) The structure of turbulent boundary layers. J Fluid Mech 30:741-773

Kushwaha A, Park JS, Graham MD (2017) Temporal and spatial intermittencies within channel flow turbulence near transition. Phys Rev Fluids 2(024603):1-30

Moser RD, Kim J, Mansour N (1999) Direct numerical simulation of turbulent channel flow up to Re $=590$. Phys Fluids 11:943-945

Owolabi BE, Dennis DJC, Poole RJ (2017) Turbulent drag reduction by polymer additives in parallel-shear flows. J Fluid Mech 827:R4-1-R4-12

Park JS, Graham MD (2015) Exact coherent states and connections to turbulent dynamics in minimal channel flow. J Fluid Mech 782:430-454

Patel VC, Head MR (1969) Some observations on skin friction and velocity profiles in fully developed pipe and channel flows. J Fluid Mech 38:181-201

Quadrio M (2011) Drag reduction in turbulent boundary layers by inplane wall motion. Philos Trans R Soc A 369:1428-1442

Robinson SK (1991) Coherent motions in the turbulent boundary layer. Ann Rev Fluid Mech 23:601-639

Tropea C (1995) Laser Doppler aneometry: recent developments and future challenges. Meas Sci Technol 6:605-619

Tsukahara T, Kawaguchi Y, Kawamura H, Tillmark N, Alfredsson PH (2010) Turbulent stripe in transitional channel flow with/without system rotation. In: Seventh IUTAM symposium on laminar-turbulent transition, IUTAM Bookseries 18

Virk PS (1975) Drag reduction fundamentals. AIChE J 21(4):625-656

Virk PS, Mickley HS, Smith KA (1970) The ultimate asymptote and mean flow structure in Toms' phenomena. J Appl Mech $37: 488-493$

Waleffe F (1998) Three-dimensional coherent states in plane shear flows. Phys Rev Lett 81(19):4140-4143

Waleffe F (2003) Homotopy of exact coherent structures in plane shear flows. Phys Fluids 15:1517-1534

Wang SN, Shekar A, Graham MD (2017) Spatiotemporal dynamics of viscoelastic turbulence in transitional channel flow. J Non Newt Fluid Mech 244:104-122

Wedin H, Kerswell RR (2004) Exact coherent structures in pipe flow: travelling wave solutions. J Fluid Mech 508:333-371

Wei T, Willmarth WW (1989) Reynolds-number effects on the structure of a turbulent channel flow. J Fluid Mech 204:57-95

Westerweel J (1997) Fundamentals of digital particle image velocimetry. Meas Sci Technol 8:1379

Whalley RD, Choi KS (2014) Turbulent boundary-layer control with plasma spanwise travelling waves. Exp Fluids 55(1796):1-16 
Whalley RD, Park JS, Kushwaha A, Dennis DJC, Graham MD, Poole RJ (2017) Low-drag events in transitional wall-bounded turbulence. Phys Rev Fluids 2(034602):1-9

Willis AP, Peixinho J, Kerswell RR, Mullin T (2008) Experimental and theoretical progress in pipe flow transition. Philos Trans $\mathrm{R}$ Soc A 366:2671-2684

Xi L, Graham MD (2010a) Active and hibernating turbulence in minimal channel flow of Newtonian and polymeric fluids. Phys Rev Lett 104(218):301

Xi L, Graham MD (2010b) Turbulent drag reduction and multistage transitions in viscoelastic minimal flow units. J Fluid Mech 647:421-452
Xi L, Graham MD (2012a) Dynamics on the laminar-turbulent boundary and the origin of the maximum drag reduction asymptote. Phys Rev Lett 108(028301):1-5

Xi L, Graham MD (2012b) Intermittent dynamics of turbulence hibernation in Newtonian and viscoelastic minimal channel flows. J Fluid Mech 693:433-472

Publisher's Note Springer Nature remains neutral with regard to jurisdictional claims in published maps and institutional affiliations. 